\title{
Unveiling the Ionic Diels-Alder Reactions within the Molecular Electron Density Theory
}

\author{
Luis R. Domingo *(D), Mar Ríos-Gutiérrez and María José Aurell \\ Department of Organic Chemistry, University of Valencia, Dr. Moliner 50, E-46100 Valencia, Spain; \\ M.Mar.Rios@uv.es (M.R.-G.); maria.j.aurell@uv.es (M.J.A.) \\ * Correspondence: domingo@utopia.uv.es
}

check for updates

Citation: Domingo, L.R.;

Ríos-Gutiérrez, M.; Aurell, M.J.

Unveiling the Ionic Diels-Alder Reactions within the Molecular

Electron Density Theory. Molecules 2021, 26, 3638. https://doi.org/

$10.3390 /$ molecules 26123638

Academic Editor: Israel Fernández

Received: 26 May 2021

Accepted: 11 June 2021

Published: 14 June 2021

Publisher's Note: MDPI stays neutral with regard to jurisdictional claims in published maps and institutional affiliations.

Copyright: (c) 2021 by the authors. Licensee MDPI, Basel, Switzerland. This article is an open access article distributed under the terms and conditions of the Creative Commons Attribution (CC BY) license (https:// creativecommons.org/licenses/by/ $4.0 /)$.

\begin{abstract}
The ionic Diels-Alder (I-DA) reactions of a series of six iminium cations with cyclopentadiene have been studied within the Molecular Electron Density Theory (MEDT). The superelectrophilic character of iminium cations, $\omega>8.20 \mathrm{eV}$, accounts for the high reactivity of these species participating in I-DA reactions. The activation energies are found to be between 13 and $20 \mathrm{kcal} \cdot \mathrm{mol}^{-1}$ lower in energy than those associated with the corresponding Diels-Alder (DA) reactions of neutral imines. These reactions are low endo selective as a consequence of the cationic character of the TSs, but highly regioselective. Solvents have poor effects on the relative energies, and an unappreciable effect on the geometries. In acetonitrile, the activation energies increase slightly as a consequence of the better solvation of the iminium cations than the cationic TSs. Electron localization function (ELF) topological analysis of the bonding changes along the I-DA reactions shows that they are very similar to those in polar DA reactions. The present MEDT study establishes that the global electron density transfer (GEDT) taking place at the TSs of I-DA reactions, and not steric (Pauli) repulsions such as have been recently proposed, are responsible for the features of these types of DA reactions.
\end{abstract}

Keywords: ionic Diels-Alder reactions; Molecular Electron Density Theory; iminium cations; superelectrophiles; global electron density transfer; asynchronicity

\section{Introduction}

The Diels-Alder (DA) reaction between a conjugated diene and an ethylene to yield a cyclohexene, reported for the first time by Diels and Alder in 1928 [1], is one of the most studied organic reactions from a synthetic as well as a theoretical viewpoint (see Scheme 1) [2,3].

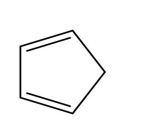

1<smiles>O=C1C=CC(=O)O1</smiles>

2

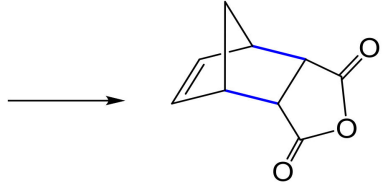

3
Scheme 1. DA reaction between Cp 1 and maleic anhydride 2 reported in 1928 by Diels and Alder.

The mechanism of the DA reaction has been the subject of many theoretical studies. The most accepted one is the "pericyclic mechanism" proposed by Woodward and Hoffman in 1969 [4], and further supported by Houk in 1995 [5]. The "pericyclic mechanism" concept refers as the electron density changes along the DA reactions, but this mechanism was not quantum chemically proved until 2002, when a bonding evolution theory [6] (BET) study of the DA reaction between butadiene 4 and ethylene 5 showed that the changes in electron density take place sequentially along a symmetrical plane (see Scheme 2) [7,8]. 


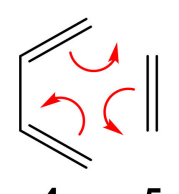

(a)

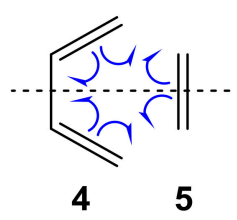

(b)

Scheme 2. Changes in electron density in the DA reaction between butadiene 4 and ethylene 5: (a) those proposed in the pericyclic mechanism [4]; (b) those resulting from a BET analysis [8].

After an exhaustive study of organic reactions carried out over 20 years, in 2016, Domingo proposed the Molecular Electron Density Theory [9] (MEDT) for the study of the organic reactivity. MEDT contrasts against any reactivity model based on the analysis of molecule orbitals (MOs) such as the proposed frontier molecular orbital (FMO) theory [10], enabled rejections of both the "pericyclic mechanism" [8] proposed by Woodward and Hoffman and the "aromatic transition state structures (TSs)" proposed by Dewar [11].

An exhaustive theoretical study on experimental DA reactions established the definitive role of the global electron density transfer [12] (GEDT) taking place at the TSs in the feasibility of DA reactions $[13,14]$. In 2009, this finding established the mechanism of polar DA (P-DA) reactions [15], in which the favorable nucleophilic/electrophilic interactions taking place at the TSs are responsible for the feasibility of the reaction. The very good correlation found between the GEDT and the activation barriers for the DA reactions of Cp 1 with a series of 12 substituted ethylenes, including iminium cation 6, enabled the classification of the DA reactions in non-polar DA (N-DA), which do not take place easily experimentally, P-DA reactions, and ionic DA (I-DA) reactions, in which one of the two reagents is an ionic species (see Figure 1) [15]. In this sense, analysis of the electrophilicity [16] $\omega$ and nucleophilicity [17] $N$ indices, defined within the conceptual DFT $[18,19]$ (CDFT), has becomes a powerful tool for experimentalist organic chemists to easily study DA reactions [15].

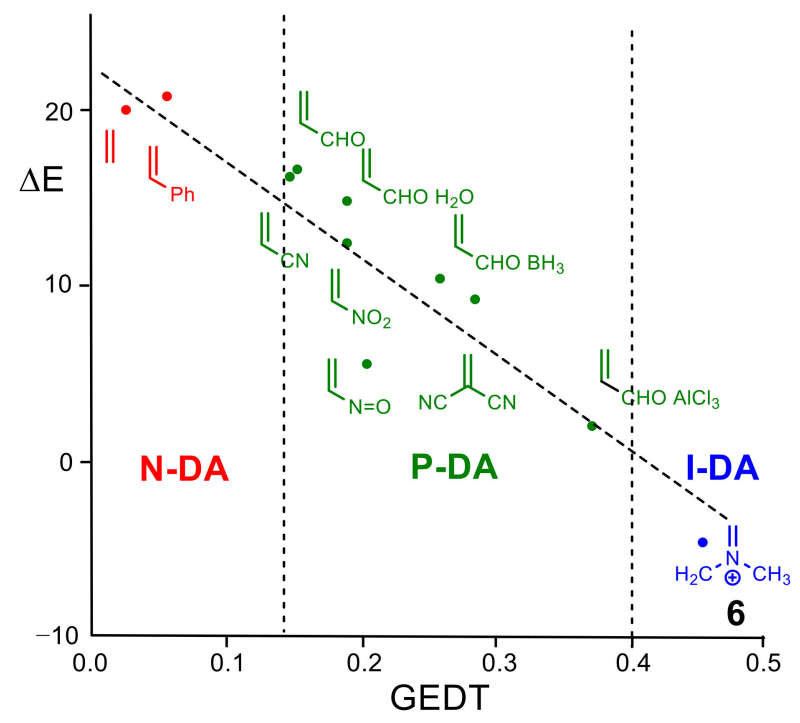

Figure 1. Plot of the activation barriers $\left(\Delta \mathrm{E}\right.$ in $\left.\mathrm{kcal} \cdot \mathrm{mol}^{-1}\right)$ vs. the GEDT, e, $R^{2}=0.89$, for the DA reactions of $C p 1$ with the substituted ethylene series of an increased electrophilic character. The classification of the DA reactions, based on the GEDT, is included.

Iminium cation 6 was found to be the most reactive species, showing the highest electrophilicity $\omega$ index, $8.24 \mathrm{eV}$. This very high value, which classifies it as a superelectrophile [20,21], is a consequence of its cationic nature. Although I-DA reactions can be understood as the extreme of P-DA reactions, the different behaviors of the TSs involved in 
I-DA reactions, with respect to those of P-DA reactions, demanded their classification as I-DA reactions (see Figure 1) [15].

Many I-DA reactions have been described in the literature; however, the majority of them involves cationic species such as cationic derivatives of imines. This behavior is a consequence of the fact that whereas superelectrophilic species react with poor or marginal nucleophiles, supernucleophilic species, such as anionic species, do no react effectively with poor or marginal electrophiles. Thus, Sauer et al. studied the DA reactions between several salts of iminium cation 6 and substituted butadienes 7 , both experimentally and theoretically using AM1 semiempirical calculations (see Scheme 3) [22]. These authors suggested that the DA reactions of iminium cation 6 with the acyclic 1,3-butadienes 7 proceeded via transition state structures (TSs) that closely resemble those yielding intermediate allyl cations. Only in the case of reaction with $\mathrm{Cp} \mathbf{1}$ did these authors conclude that the reaction takes place along a pericyclic TS [22].

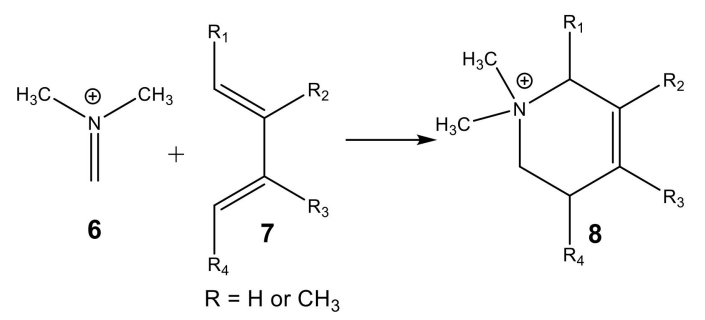

Scheme 3. I-DA reactions experimentally and theoretically studied by Sauer et al. [22].

Andersson et al. explored the DA reaction between Cp $\mathbf{1}$ and a family of chiral imines containing a second nitrogen atom at a conjugated position, because in a strong acid medium, the protonation of this nitrogen atom might fulfil the role of an electronwithdrawing group [23]. Thus, when imine 9 was treated with Cp $\mathbf{1}$ under strong acid conditions at $-78{ }^{\circ} \mathrm{C}$, a highly stereoselective cycloaddition took place, giving the pyridinesubstituted aza-norbornene $\mathbf{1 0}$ in good yield (see Scheme 4). Note that this reaction is a Bronsted acid-catalyzed DA reaction of the imine $\mathbf{9}$.<smiles>C[C@H](N=Cc1ccccn1)c1ccccc1</smiles>

9<smiles>CC(N=Cc1ccccn1)c1ccccc1</smiles>

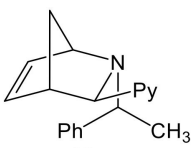

10

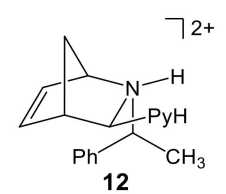

Scheme 4. I-DA reactions experimentally studied by Andersson et al. [23].

The participation of cationic species as the heterodiene or the ethylene derivative in DA reactions has been widely studied in the literature. Thus, in 2001, the I-DA reaction between iminium cation 6 and $\mathrm{Cp}$ 1, experimentally studied by Sauer [22], was studied at the B3LYP/6-31G(d) and MP2/6-31G(d) levels [24]. This study demonstrated that this I-DA reaction takes place via a one-step mechanism involving a highly asynchronous TS with a high GEDT. A complete analysis of the features of the TS indicated that this I-DA reaction can be characterized by the nucleophilic attack of $\mathrm{Cp} \mathbf{1}$ on the carbon of the electron-poor iminium cation 6 instead of a "pericyclic process". The concomitant ring closure affords the final cycloadduct without the participation of any allyl cation intermediate, via a non- 
concerted two-stage one-step mechanism [25]. Consequently, iminium cation $\mathbf{6}$ and Cp 1 behave as an electrophile and nucleophile, respectively, instead of as dienophile and diene.

The I-DA reaction of $\mathrm{CP} 1$ with diprotonated imine 11, experimentally reported by Andersson [23], was theoretically studied using HF/6-31G(d) and B3LYP/6-31G(d) methods [26]. This I-DA reaction takes place along a two-step mechanism. The first step corresponds to the nucleophilic attack of $\mathrm{Cp} \mathbf{1}$ on the carbon of the iminium cation group to produce an acyclic cation intermediate, while the second step is associated with the ring closure of this intermediate along the formation of the second $\mathrm{C}-\mathrm{N}$ single bond, yielding the final cycloadduct 12. Protonation of both pyridine and imine nitrogen atoms strongly increases the electrophilicity of the imine, and the I-DA reaction takes place along a favorable inverted energy profile, allowing the justification of the large acceleration of the reaction in a strong acid medium [23]. The inclusion of solvent effects yielded a negligible variation of the energy profile.

In 2014, Domingo performed a quantum chemical topological analysis of the C-C bond formation in organic reactions involving cationic species [27]. To this end, the I-DA reaction between the iminium cation $\mathbf{6}$ and $\mathrm{Cp} \mathbf{1}$ was chosen as a computational model. The BET analysis showed that $\mathrm{C}-\mathrm{C}$ bond formation begins at the $\mathrm{C}-\mathrm{C}$ distance of $1.96 \AA$ via a C-to-C pseudoradical coupling between the most electrophilic center of the iminium cation and one of the two most nucleophilic centers of Cp. Interestingly, this pattern for the formation of the $\mathrm{C}-\mathrm{C}$ single bond was identical to that found in the N-DA reaction between butadiene 4 and ethylene 5 [12]. Analysis of the Parr functions [28] enabled characterization of the most electrophilic and nucleophilic centers of the reagents participating in this I-DA reaction.

Very recently, Bickelhaup et al. studied the origin of rate enhancement and asynchronicity in iminium-catalyzed DA reactions using an energy decomposition analysis [29]. Through their activation strain model (ASM) [30] and Kohn-Sham molecular orbital [31] analyses, these authors suggested that "the iminium catalysts enhance the reactivity by reducing the steric (Pauli) repulsion between the diene and dienophile, which originates from both a more asynchronous reaction mode and a more significant polarization of the $\pi$-system away from the incoming diene compared to aldehyde and imine analogs". They concluded that "the driving force behind the asynchronicity of these DA reactions is the relief of destabilizing steric (Pauli) repulsion and not the orbital interaction between the terminal carbon of the dienophile and the diene" [29].

The asynchronicity in cycloaddition reactions has been widely studied within the polar reaction model [13-15]. P-DA reactions involving symmetric electrophilic ethylenes such as anhydride maleic or tetracyanoethylene take place through synchronous TSs [14], whereas the use of non-symmetric electrophilic ethylenes such as nitroethylene or 1,1dicyanoethylene yields highly asynchronous TSs [13-15,32] These studies emphasized that along an asynchronous single bond formation process, the more favorable two-center interaction begins at the most electrophilic and nucleophilic centers of the two interacting molecules. A good linear correlation between the GEDT and the asynchronicity at eight of the TSs associated with the P-DA and I-DA reactions given in Figure 1 can be established, $R^{2}=0.95$ (see Figure S1 in Supplementary Material). As shown, the asynchronicity increases with the increase in the polar character of the reaction, i.e., the increase in the GEDT at the TSs [13-15,32]. The stronger the nucleophilic/electrophilic interactions at the TSs involving non-symmetric electrophiles, the higher the asynchronicity. As a consequence, I-DA reactions can take place through a non-concerted two-stage one-step mechanism [25] via a highly asymmetric TS [24], even though a two-step mechanism involves a cation intermediate [26]. The increase in the asynchronicity enhances the distance between the ends of the two interacting frameworks, the iminium cation, and the diene (see TS-III in Figure 2), and consequently, decreases the steric (Pauli) repulsions. Consequently, the decrease in steric (Pauli) repulsions is a consequence of the increase in the asynchronicity, and not vice versa; asynchronicity in P-DA and I-DA reactions depends on the GEDT taking place at the TSs, which depends on the electrophilic/nucleophilic nature of the reagents. 


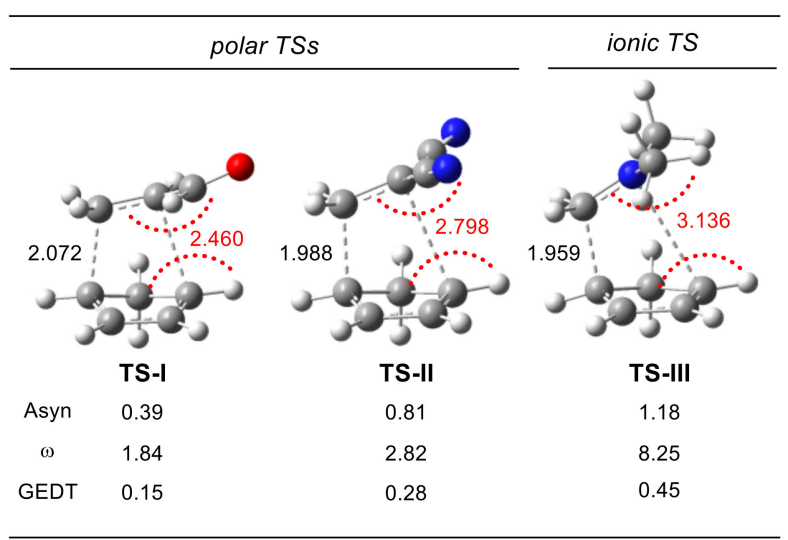

Figure 2. B3LYP/6-32G(d) Asynchronicity, Asyn = d1-d2 in Angstroms, $\AA$, electrophilicity $\omega$ index of the ethylene, in eV, and GEDT, in e, at the TSs involved in P-DA reactions, TS-I and TS-II, and in I-DA reaction, TS-III.

Although I-DA reaction can be viewed as the extreme of P-DA reactions [15], they have many behaviors different from P-DA reactions, i.e., I-DA reactions are poorly endo selective, and polar solvents cause a contrary effect in P-DA reactions. Herein, a complete MEDT study of I-DA reactions involving cationic species is presented. To this end, the I-DA reactions of six iminium cations, 6, 15-19, with Cp 1 were selected as computational models (see Scheme 5). It is interesting to remark that protonated imines $\mathbf{1 5}$ and $\mathbf{1 7}$ can be considered as species participating in acid Bronsted-catalyzed DA reactions of imines 13 and 14 [23].

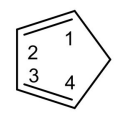

$\mathrm{Cp}, 1$<smiles>C=[N+](C)[15NH2]</smiles><smiles>C=CC=[N+]([O-])O</smiles>

17

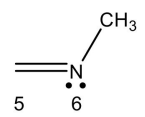

13

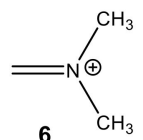

6

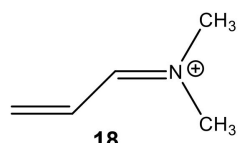

18

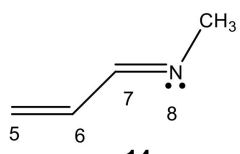

14
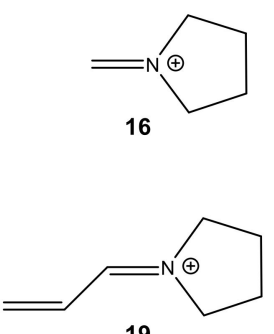

Scheme 5. Structures of the species involved in the P-D reactions of Cp $\mathbf{1}$ with imines $\mathbf{1 3}$ and 14, and the I-DA reactions with iminium cation 6, 15-19.

The present MEDT study responds to the following questions: (i) What is the origin of the high acceleration and asynchronicity found in I-DA reactions?; (ii) What is the origin of the low endo selectivity in contrast with the high endo selectivity found in P-DA reactions?; and (iii) What is the role of the solvent polarity in these I-PA reactions?

\section{Results and Discussions}

The present MEDT study has been divided into three parts: (i) first, a study of the electronic structure and reactivity at the ground state (GS) of the reagents is performed; (ii) in the second part, a study of the potential energy surface of the I-DA reactions of iminium cations 6, 15-19 with Cp 1 is carried out; and finally, (iii) in the third part, a topological analysis of the bonding changes along the I-DA reactions is presented. The origin of the regioselectivity in I-DA reactions and asynchronicity in bond formation at the TSs is analyzed on the basis of changes in electron density. 


\subsection{Study of the Electronic Structure and Reactivity at the Ground State of the Reagents}

\subsubsection{Analysis of the Electronic Structure of Imines and Iminium Cations}

In order to obtain information about how the formation of the iminium cations modifies the electronic structure of imines, electron localization function [33] (ELF) and natural population analysis [34,35] (NPA) assays of imines $\mathbf{1 3}$ and 14, and iminium cations $\mathbf{6}$ and $\mathbf{1 8}$, was performed. The ELF valence basin localization domains, the ELF-based Lewis-like structures, and natural atomic charges are presented in Figure 3.
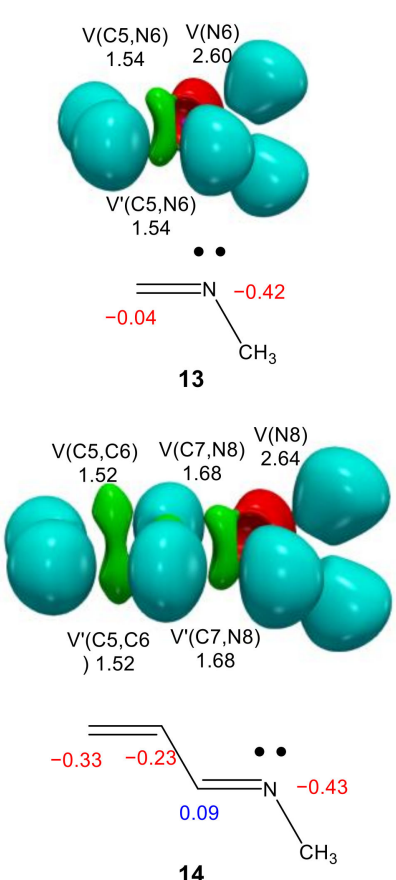
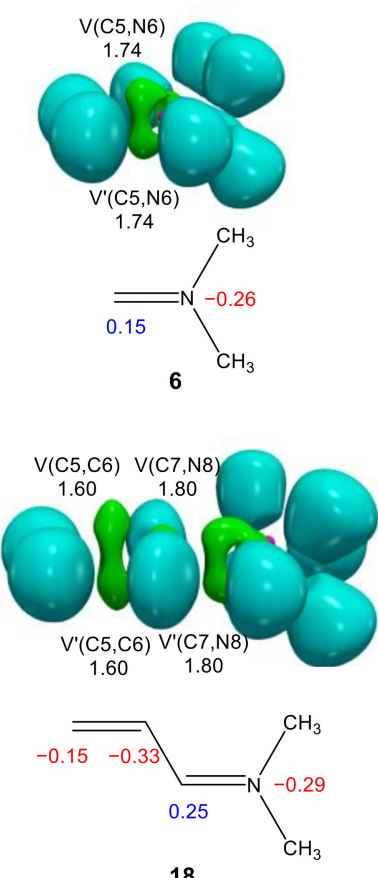

18

Figure 3. $\omega B 97 X D / 6-311 G(d, p)$ localization domains (isosurface value $=0.75$ ): blue color is used to show protonated basins, red color for the monosynaptic basins, green color for the disynaptic basins and magenta color for core basins, ELF based Lewis-like structures, and natural atomic charges, in average number of electrons e, blue color is used for positive charges and red color for negative charges, of imines 13 and 14, and iminium cations 6 and 18.

ELF of imine 13 shows the presence of two disynaptic basins, $\mathrm{V}(\mathrm{C} 5, \mathrm{~N} 6)$ and $\mathrm{V}^{\prime}(\mathrm{C} 5, \mathrm{~N} 6)$, integrating a total of $3.08 \mathrm{e}$, one $\mathrm{V}(\mathrm{N} 6)$ monosynaptic basin integrating $2.60 \mathrm{e}$, and one $\mathrm{V}(\mathrm{N} 6, \mathrm{C}(\mathrm{Me}))$ disynaptic basin, integrating $1.74 \mathrm{e}$. The two disynaptic basins are associated with the underpopulated $\mathrm{C} 5=\mathrm{N} 6$ double bond, and the monosynaptic basin is associated with the non-bonding electron density of the N6 nitrogen. ELF of conjugated imine $\mathbf{1 4}$ shows the presence of two disynaptic basins, $\mathrm{V}(\mathrm{C} 5, \mathrm{C} 6)$ and $\mathrm{V}^{\prime}(\mathrm{C} 5, \mathrm{C} 6)$, integrating a total of $3.04 \mathrm{e}$, one $\mathrm{V}(\mathrm{C} 6, \mathrm{C} 7)$ disynaptic basins integrating $2.22 \mathrm{e}$, two disynaptic basins, $\mathrm{V}(\mathrm{C} 7, \mathrm{~N} 8)$ and $\mathrm{V}^{\prime}(\mathrm{C} 7, \mathrm{~N} 8)$, integrating a total of $3.36 \mathrm{e}$, and one $\mathrm{V}(\mathrm{N} 8)$ monosynaptic basin, integrating 2.64 e. The two $\mathrm{V}(\mathrm{C} 5, \mathrm{C} 6)$ and $\mathrm{V}^{\prime}(\mathrm{C} 5, \mathrm{C} 6)$ disynaptic basins are associated with the underpopulated $\mathrm{C} 5=\mathrm{C} 6$ double bond, the $\mathrm{V}(\mathrm{C} 6, \mathrm{C} 7)$ disynaptic basin is associated with the overpopulated $\mathrm{C} 6-\mathrm{C} 7$ single bond, the $\mathrm{V}(\mathrm{C} 7, \mathrm{~N} 8)$ and $\mathrm{V}^{\prime}(\mathrm{C} 7, \mathrm{~N} 8)$ disynaptic basins are associated with the underpopulated $\mathrm{C} 7=\mathrm{N} 8$ double bond, and the monosynaptic basin is associated with the non-bonding electron density of the $\mathrm{N} 8$ nitrogen. In addition, one $\mathrm{V}(\mathrm{N} 8, \mathrm{C}(\mathrm{Me}))$ disynaptic basin, integrating $1.75 \mathrm{e}$, associated with the N8-C(Me) single bond is observed.

ELF of iminium cation 6 shows the presence of two disynaptic basins, $\mathrm{V}(\mathrm{C} 5, \mathrm{~N} 6)$ and $\mathrm{V}^{\prime}(\mathrm{C} 5, \mathrm{~N} 6)$, integrating a total of $3.48 \mathrm{e}$, and two disynaptic basins, $\mathrm{V}(\mathrm{N} 6, \mathrm{C}(\mathrm{Me}))$ and $\mathrm{V}(\mathrm{N} 6$, $\mathrm{C}^{\prime}(\mathrm{Me})$ ), integrating $1.92 \mathrm{e}$ each. ELF of conjugated iminium cation 18 shows the presence of two disynaptic basins, $\mathrm{V}(\mathrm{C} 5, \mathrm{C} 6)$ and $\mathrm{V}^{\prime}(\mathrm{C} 5, \mathrm{C} 6)$ integrating a total of $3.20 \mathrm{e}$, one $\mathrm{V}(\mathrm{C} 6, \mathrm{C} 7)$ disynaptic basins integrating $2.22 \mathrm{e}$, and two disynaptic basins, $\mathrm{V}(\mathrm{C} 7, \mathrm{~N} 8)$ and $\mathrm{V}^{\prime}(\mathrm{C} 7, \mathrm{~N} 8)$, 
integrating a total of $3.60 \mathrm{e}$. In addition, two disynaptic basins, $\mathrm{V}(\mathrm{N} 8, \mathrm{C}(\mathrm{Me}))$ and $\mathrm{V}(\mathrm{N} 8$, $\mathrm{C}^{\prime}(\mathrm{Me})$ ), integrating 1.88 e each, are observed.

On the other hand, ELF of Cp 1, not represented in Figure 3, shows the presence of two disynaptic basins, $\mathrm{V}(\mathrm{C} 1, \mathrm{C} 2)$ and $\mathrm{V}^{\prime}(\mathrm{C} 1, \mathrm{C} 2)$, integrating a total of $3.38 \mathrm{e}$, one $\mathrm{V}(\mathrm{C} 2, \mathrm{C} 3)$ disynaptic basin, integrating a total of $2.21 \mathrm{e}$, and two disynaptic basins, $\mathrm{V}(\mathrm{C} 3, \mathrm{C} 4)$ and $\mathrm{V}^{\prime}(\mathrm{C} 3, \mathrm{C} 4)$, integrating a total of $3.38 \mathrm{e}$. The $\mathrm{C} 1=\mathrm{C} 2-\mathrm{C} 3=\mathrm{C} 4$ bonding region accumulated a total of $8.97 \mathrm{e}$.

The natural atomic charges are given in Figure 3. At iminium cation 6, while the C5 carbon is positively charged by $0.15 \mathrm{e}$, the N6 nitrogen is negatively charged by $0.26 \mathrm{e}$. On the other hand, at conjugated iminium 18, while the $\mathrm{C} 5$ and $\mathrm{C} 6$ carbons are negatively charged by 0.15 and $0.33 \mathrm{e}$, respectively, the $\mathrm{C} 7$ carbon is slightly positively charged by $0.25 \mathrm{e}$; the N8 nitrogen is negatively charged by $0.29 \mathrm{e}$.

Some interesting conclusions can be drawn from the analysis of the electronic structure of imines 13 and 14 and iminium cations 6 and 18: (i) ELF of the $C=C$ and $C=N$ double bonds of imines $\mathbf{1 3}$ and $\mathbf{1 4}$ showed a depopulation of these bonding regions as a consequence of the polarization of the molecular electron density towards the $\mathrm{N}$ nitrogen; note that the non-bonding region of the nitrogen integrated more than $2.60 \mathrm{e}$; (ii) the populations of these bonding regions were higher at iminium cations $\mathbf{6}$ and $\mathbf{1 8}$ than at imines 13 and 14. This finding is a consequence of the formation of the new $\mathrm{N}-\mathrm{C}(\mathrm{Me})$ single bonds, which are populated by less charge of $1.92 \mathrm{e}$. Consequently, the excess of the non-bonding nitrogen electron density of imines $\mathbf{1 3}$ and $\mathbf{1 4}$ is redistributed towards the $\mathrm{C}-\mathrm{C}$ and $\mathrm{C}-\mathrm{N}$ bonding regions of $\mathbf{6}$ and 18; (iii) the nitrogen centers of the iminium cations 6 and 18 are negatively charged, a behavior against the positive charge represented at the Lewis structures of iminium cations. This behavior is a consequence of the fact that in organic cationic species, the hydrogens are the centers which mainly support the positive charge; (iv) analysis of the electronic structures of the conjugated imine 14 and iminium cation 18 shows that the different substitution on the $C 5=C 6$ double bonds of these species does not substantially modify the electronic structure of the ethylene framework participating in these DA reactions; and finally, (v) at the most electrophilic iminium cation 18, the $\beta$-conjugated C5 carbon, which supports a negative charge of $-0.15 \mathrm{e}$, is the most electrophilic center of this cationic species; see analysis of the electrophilic Parr functions. This finding supports the earlier observation that the electrophilicity and nucleophilicity cannot be related only with charge distribution at the GS of the molecules, but with the propensity of changes on electron density resulting from the GEDT along a polar reaction, which is anticipated by the analysis of the Parr functions [28].

\subsubsection{Analysis of the CDFT Reactivity Indices at the GS of the Reagents}

Many studied devoted to cycloaddition reactions have proven that the analysis of the global and local reactivity indices defined within the CDFT $[18,19]$ is a powerful tool to understand polar reactions [36,37]. The CDFT indices were obtained at the B3LYP/6-31G(d) computational level because they were used to define the scales of electrophilicity and nucleophilicity [19]. The global reactivity indices of imines 13 and 14, iminium cations $\mathbf{6}$, 15-19, and Cps 1 and 20 are collected in Table 1.

The electronic chemical potentials $[18,38] \mu$ of imines, $\mu=-3.46(\mathbf{1 3})$ and $-3.91(\mathbf{1 4}) \mathrm{eV}$, are closer to that of $\mathrm{Cp} 1, \mu=-3.01 \mathrm{eV}$, indicating that the corresponding HDA reactions will have non-polar characteristics and high activation energies. On the other hand, the six iminium cations present very low electronic chemical potential $\mu$ values, between -9.53 (18) and -11.83 (15) eV. Consequently, the corresponding I-DA reactions will experience a high GEDT and very low activation energies. 
Table 1. B3LYP/6-31G(d) Global reactivity indices, the electronic chemical potential, $\mu$, chemical hardness, $\eta$, electrophilicity, $\omega$, and nucleophilicity, $N$, in eV, of imines 8 and 9, iminium cations 1-6, and $\mathrm{Cp} 7$.

\begin{tabular}{ccccc}
\hline & $\boldsymbol{\mu}$ & $\boldsymbol{\eta}$ & $\boldsymbol{\omega}$ & $\boldsymbol{N}$ \\
\hline $\mathbf{1 7}$ & -10.11 & 5.36 & 9.53 & -3.67 \\
$\mathbf{1 5}$ & -11.83 & 7.80 & 8.97 & -6.61 \\
$\mathbf{1 9}$ & -9.76 & 5.32 & 8.95 & -3.30 \\
$\mathbf{1 8}$ & -9.53 & 5.22 & 8.71 & -3.02 \\
$\mathbf{6}$ & -11.18 & 7.56 & 8.26 & -5.84 \\
$\mathbf{1 6}$ & -10.51 & 6.73 & 8.20 & -4.75 \\
$\mathbf{1 4}$ & -3.91 & 5.84 & 1.31 & 2.30 \\
$\mathbf{1 3}$ & -3.46 & 6.80 & 0.88 & 2.26 \\
Cp $\mathbf{1}$ & -3.01 & 5.48 & 0.83 & 3.37 \\
CpMe $\mathbf{2 0}$ & -2.84 & 5.31 & 0.76 & 3.62 \\
\hline
\end{tabular}

The electrophilicity $\omega$ indexes [16] of imines $\mathbf{1 3}$ and $\mathbf{1 4}$ are 0.88 and $1.31 \mathrm{eV}$, respectively, being classified as marginal electrophiles, whereas the nucleophilicity $N$ values [17], 2.26 and $2.30 \mathrm{eV}$, also classify them as moderate nucleophiles. The presence of the electronegative N8 nitrogen in the heterodiene $\mathbf{1 4}$ decreases its nucleophilic character with respect to that of 1,3-butadiene, $N=2.93$ e. Consequently, imines 13 and 14 will not participate, either as ethylene or as diene in P-DA reactions.

The electrophilicity $\omega$ index of iminium cations, between 8.20 (16) and 9.53 (17) eV, classifies them as strong electrophiles. These high values, higher than $4.0 \mathrm{eV}$, classify them as superelectrophiles [21]. Consequently, they will participate in I-DA reactions with unappreciable activation energies. All iminium cations present negative nucleophilicity $N$ indices.

The electrophilicity $\omega$ and nucleophilicity $N$ indices of $C p 1,0.83$ and $3.37 \mathrm{eV}$, respectively, classify it as a moderate electrophile and a strong nucleophile. The inclusion of an electron-releasing methyl group on the $\mathrm{C} 1$ carbon of $\mathrm{Cp} \mathbf{1}$ decreases the electrophilicity $\omega$ index of CpMe 20 to $0.76 \mathrm{eV}$ and increases its nucleophilicity $N$ index to $3.62 \mathrm{eV}$, thus classifying it as a marginal electrophile and a strong nucleophile.

From the electrophilicity $\omega$ indices of the iminium cations given in Table 1, some interesting conclusions can be drawn: (i) the series of the conjugated iminium cations 17-19 are more electrophilic than iminium cations $\mathbf{6}$ and 16. In spite of that, the latter have less electronic chemical potential $\mu$; the softer character of the former makes them more electrophilic. Note that the softness, $S$, is the inverse of the hardness, $\eta, S=1 / \eta$; (ii) the protonated iminium cations are more electrophilic than the dialkyl derivative cations, due to the electron-releasing character of the $\mathrm{CH}_{3}$ and $\mathrm{CH}_{2}$ groups; and finally, (iii) the two cyclic iminium cations have different behaviors. The dimethyl derivative $\mathbf{6}$ is more electrophilic than cyclic 16, whereas cyclic iminium cation 19 is more electrophilic than the dimethyl derivative $\mathbf{1 8 .}$

DA reactions involving non-symmetric electrophilic compounds take place via asynchronous TSs. The most favorable two-center interactions between the two interacting species in the polar process takes place between the most electrophilic center of the electrophile, and the most nucleophilic center of the nucleophile [39]. In this context, the electrophilic $P_{k}^{+}$and nucleophilic $P_{k}^{-}$Parr functions [28] derived from the excess of spin electron density reached via the GEDT [12] process from the nucleophile toward the electrophile have been shown to be one of the most accurate and insightful tools for the study of local reactivity in polar and ionic processes. The $\mathrm{C} 1$ and $\mathrm{C} 4$ carbons of $\mathrm{Cp} 7$ are the most nucleophilic centers of this diene. In order to explain the asynchronicity in the $\mathrm{C}-\mathrm{C}$ and $\mathrm{C}-\mathrm{N}(\mathrm{C})$ bond formation, the electrophilic $P_{k}^{+}$functions of imines $\mathbf{1 3}$ and $\mathbf{1 4}$, and dimethyl iminium cations 6 and 18, were analyzed. The corresponding values are given in Figure 4. 


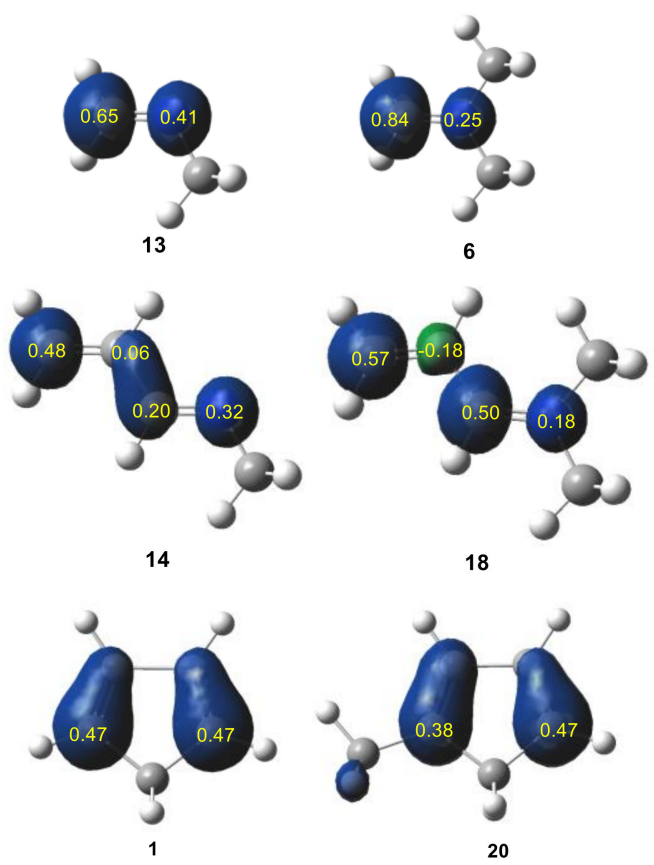

Figure 4. Three-dimensional representations of the Mulliken atomic spin densities of the radical anions of imines $\mathbf{1 3}$ and 14, neutral radicals 6 and 18, the radical cations of Cp 1 and CpMe 20, and the electrophilic $P_{k}^{+}$and nucleophilic $P_{k}^{-}$Parr functions.

As expected, the C5 carbon of imine 13 and iminium cation $6, P_{k}^{+}=0.65$ and 0.84 , respectively, are more electrophilically activated than the N6 nitrogen, $P_{k}^{+}=0.41$ and 0.25 . Formation of the iminium cation 6 increases the electrophilic activation of the $\mathrm{C} 5$ carbon, indicating that the corresponding TS will be more asynchronous.

The C 5 carbon of conjugated imine $14, P_{k}^{+}=0.48$, is also the most electrophilic center of this species, following the N8 nitrogen, $P_{k}^{+}=0.32$. On the other hand, despite the strong electrophilic activation of the $\mathrm{C} 7$ carbon of iminium cation $18, P_{k}^{+}=0.50$, the $\mathrm{C} 5$ carbon remains as the most electrophilic center of this species, $P_{k}^{+}=0.57$. Note that the C6 carbon is electrophilically deactivated, at -0.18 , indicating that the corresponding TS will be highly asynchronous.

Analysis of the electrophilic $P_{k}^{+}$Parr functions of these species indicates that the most favorable two-center interactions at the corresponding TSs will take place between one of the two end carbons of the symmetric diene $\mathrm{Cp}$ 1, i.e., the $\mathrm{C} 1$ carbon, and the $\mathrm{C} 5$ carbons of these imine derivatives. Formation of the corresponding iminium cation increases the electrophilic activation of the $\mathrm{C} 5$ carbon with respect to the $\mathrm{N} 6$ or $\mathrm{C} 6$ centers, increasing the asynchronicity in $\mathrm{C}-\mathrm{N}(\mathrm{C})$ single bond formation.

It is interesting to remark that the electrophilic activation of the $\mathrm{C} 5$ carbon of iminium cation 18, $P_{k}^{+}=0.57$, is higher than that of the C7 carbon, $P_{k}^{+}=0.50$, even though the C5 carbon is negatively charged, $-0.15 \mathrm{e}$, whereas the $\mathrm{C} 7$ carbon is positively charged, 0.25 e (see Figure 3). This finding supports the earlier assumptions that electrophilicity is not controlled by positively charged centers [21,32].

Analysis of the nucleophilic $P_{k}^{-}$Parr functions of $\mathrm{Cp} 1$ indicates that the $\mathrm{C} 1$ and $\mathrm{C} 4$ are symmetrically activated. These carbons are the most nucleophilic center of $\mathrm{Cp}, P_{k}^{-}=0.47$. The inclusion of an ER-Me at the C1 carbon does not modify the nucleophilic $P_{k}^{-}$Parr function at the C4 carbon, $P_{k}^{-}=0.47$, but relocates the nucleophilic $P_{k}^{-}$Parr function at the $\mathrm{C} 1$ and $\mathrm{C} 2$ carbons. Consequently, the $\mathrm{C} 4$ carbon becomes the most electrophilic center of CpMe 20. 


\subsection{Study of the Potential Energy Surface of I-DA Reactions}

\subsubsection{Study of the I-DA Reactions of Iminium Cations 6, 15-19 with Cp 1}

For the symmetric iminium cations 6 and 16, only one reaction path is feasible, whereas for the non-symmetric iminium cations 15, 17, 18 and 19, two stereoisomeric reaction paths are feasible: the endo and the exo. In addition, due to the presence of two double bonds, $\mathrm{C}=\mathrm{C}$ and $\mathrm{C}=\mathrm{N}$ in the iminium cations derived from imine 14, the chemoisomeric reaction path associated with the attack of $\mathrm{Cp} \mathbf{1}$ to the $\mathrm{C} 7=\mathrm{N} 8$ double bond of iminium cation $\mathbf{1 8}$ was also considered (see Scheme 6). The stationary points involved in the six I-DA reactions are given in Scheme 6, and the relative energies in acetonitrile are given in Table 2. Note that due to the high instability of gas phase cationic species, the relative energies are analyzed in acetonitrile. Thus, whereas acetonitrile stabilizes the neutral Cp 1 by $7.7 \mathrm{kcal} \cdot \mathrm{mol}^{-1}$, iminium cation 15 is stabilized by $53.7 \mathrm{kcal} \cdot \mathrm{mol}^{-1}$. In order to understand the behaviors of these I-DA reactions, the DA reactions of imines 13 and 14 with $C p 7$ were also studied (see Section 1 in the Supplementary Material).

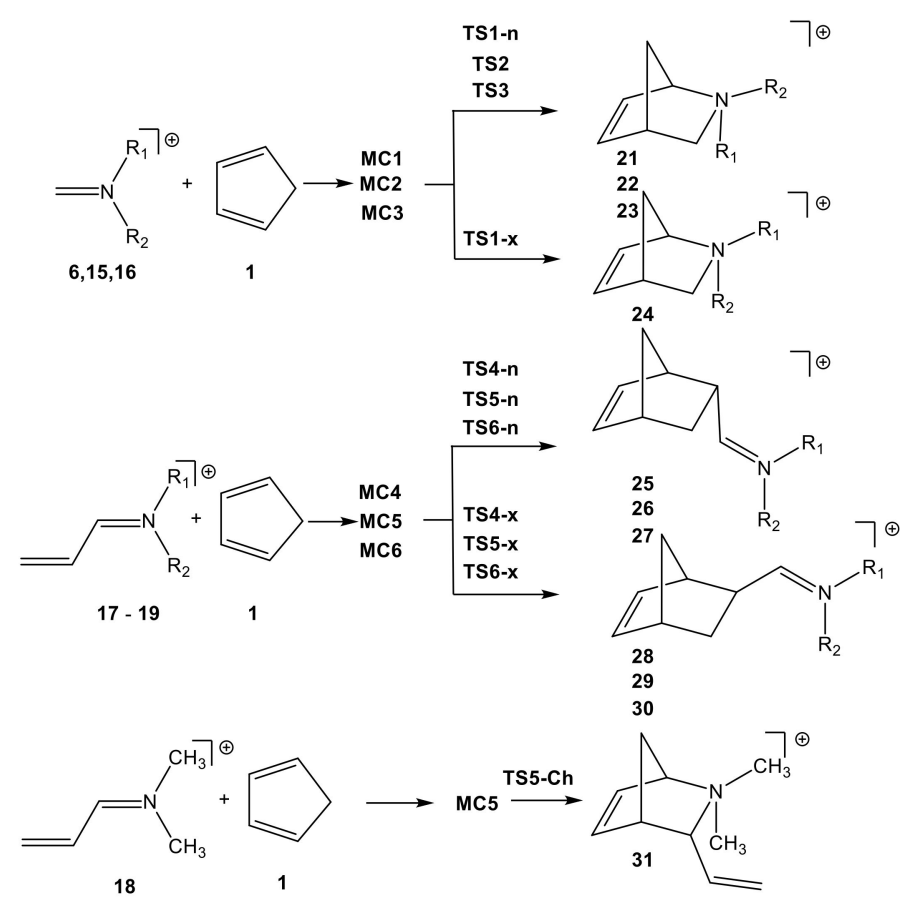

Scheme 6. I-DA reactions of iminium cations 6, 15-19 with Cp 1.

At the beginning of the reactions, a series of molecular complexes could be found. They were characterized by the presence of weak electronic interactions between $\mathrm{Cp} 1$ and the iminium cations. For each of these I-DA reactions, only the most stable MC was selected as the energy reference. The MCs were found to be between 5.6 (MC4) and 7.2 (MC3) $\mathrm{kcal} \cdot \mathrm{mol}^{-1}$ more stable than the separated reagents. The relative energies of the TSs with respect to the separated reagents associated with these I-DA reactions were found to be between -0.6 (TS1-n) and 4.4 (TS4-x) $\mathrm{kcal} \cdot \mathrm{mol}^{-1}$; the reactions were exothermic between 24.0 (22) and $28.4(21) \mathrm{kcal} \cdot \mathrm{mol}^{-1}$.

Some interesting conclusions can be drawn from the energy results given in Table 2: (i) the formation of MCs is an exothermic process, with energy less than 7.2 (MC3) $\mathrm{kcal} \cdot \mathrm{mol}^{-1}$. However, if the thermal correction and the entropies are considered, they become exergonic (see later); (ii) considering these MCs, the activation energies associated with these I-DA reactions were found to be between 6.0 (TS1-n) and 10.0 (TS3) $\mathrm{kcal} \cdot \mathrm{mol}^{-1}$. These activation barriers are between 13 and $20 \mathrm{kcal} \cdot \mathrm{mol}^{-1}$ lower in energy that those associated with the DA reactions of neutral imines $\mathbf{1 3}$ and $\mathbf{1 4}$ with $\mathrm{Cp} \mathbf{1}$ (see Scheme S1 in Supplementary Material); (iii) these I-DA reactions present low endo stereoselectivity; the energy differences between the endo and exo TSs are less than $1.2 \mathrm{kcal} \cdot \mathrm{mol}^{-1}$ 
(TS1-n). The endo stereoselectivity decreases with the increase in the activation energy; (iv) the reaction of Cp 1 with iminium cation 18 is completely chemoselective, as TS5Ch was observed to have an energy value $7.7 \mathrm{kcal} \cdot \mathrm{mol}^{-1}$ above TS5-n. This high energy difference enables discarding the attachment of $\mathrm{Cp} 1$ onto the $\mathrm{C} 7=\mathrm{N} 8$ double bond iminium cations 17-19; (v) these I-DA reactions are highly exothermic, higher than $24.0(\mathbf{2 2})$ $\mathrm{kcal} \cdot \mathrm{mol}^{-1}$; finally, (vi) the formation of 31 is exothermic by $15.3 \mathrm{kcal} \cdot \mathrm{mol}^{-1}$. Consequently, the attachment of $\mathrm{Cp} \mathbf{1}$ on the $\mathrm{C} 7=\mathrm{N} 8$ double bond iminium cation $\mathbf{1 8}$ is both kinetically and thermodynamically disfavored with respect to the attachment to the $\mathrm{C} 5=\mathrm{C} 6$ double bonds.

Table 2. $\omega B 97 X \mathrm{X} / 6-311 \mathrm{G}(\mathrm{d}, \mathrm{p})$ relative electronic energies in acetonitrile, $\mathrm{kcal} \cdot \mathrm{mol}^{-1}$, of the stationary points involved in the I-DA reactions of iminium cations 6, 15-19 with Cp 1.

\begin{tabular}{cccccc}
\hline & $\mathbf{1 5}$ & & $\mathbf{6}$ & & $\mathbf{1 6}$ \\
\hline MC1 & -6.6 & MC2 & -6.6 & MC3 & -7.2 \\
TS1-n & -0.6 & TS2 & 2.9 & TS3 & 2.8 \\
TS1-x & 0.6 & & & & -24.6 \\
$\mathbf{2 1}$ & -28.4 & $\mathbf{2 2}$ & -24.0 & & \\
$\mathbf{2 4}$ & -28.1 & & & & $\mathbf{1 9}$ \\
\hline & $\mathbf{1 7}$ & & $\mathbf{1 8}$ & $\mathbf{M C 6}$ & -5.8 \\
MC4 & -5.6 & MC5 & -5.6 & TS6-n & 3.7 \\
TS4-n & 3.7 & TS5-n & 3.1 & TS6-x & 4.1 \\
TS4-x & 4.4 & TS5-x & 3.6 & & 27.0 \\
& & TS5-Ch & 10.8 & $\mathbf{2 7}$ & -27.7 \\
$\mathbf{2 5}$ & -28.3 & $\mathbf{2 6}$ & -28.3 & $\mathbf{3 0}$ & \\
\hline
\end{tabular}

In P-DA reactions, the endo selectivity increases with the increase in the polar character of the reaction, resulting in the GEDT taking place at the TSs [13]. The GEDT increases the zwitterionic character of the TSs, increasing the favorable electrostatic interactions appearing between the ends of the two interacting frameworks along the endo approach mode. However, in I-DA reactions, the GEDT provokes a redistribution of the electron density; thus, positive charges appear at the two interacting frameworks, consequently presenting low endo stereoselectivity

The geometries of the more favorable endo TSs associated with these I-DA reactions are given in Figure 5. The geometries of the exo TSs are given in Figure S4 in the Supplementary Material. The distances between the C1-C5 and C4-N6 interacting centers at the TSs of the I-DA reactions of iminium cations 6, $\mathbf{1 5}$ and $\mathbf{1 6}$ in acetonitrile are: 1.995 and $2.869 \AA$ at TS1-n, 1.965 and $2.875 \AA$ at TS2, and 1.931 and $3.049 \AA$ at TS3, whereas those between the C1-C5 and C4-C6 interacting centers at the TSs of the I-DA reactions of conjugated iminium cations 17-19 are: 2.019 and 2.832 at $\AA$ TS4-n, 2.019 and 2.831 $\AA$ at TS5-n, and 2.012 and 2.819 at $\AA$ TS6-n. Some interesting conclusions can be drawn from these geometrical parameters: (i) the distances indicate that these TSs are associated with highly asynchronous single bond formation processes. They are associated with a two-center interaction between the most nucleophilic center of $\mathrm{Cp} 1$, the $\mathrm{C} 1$ carbon, the most electrophilic center of these iminium cations, and the C5 carbon, a behavior anticipated by analysis of the electrophilic Parr functions; (ii) for the two series of I-DA reactions, there is a decrease in the C1-C5 distance with the increase in the activation energy; for these series, the most favorable TS is more delayed. This finding can be understood as an application of the Hammond principle [40]; finally, (iii) comparison of the gas phase and acetonitrile geometries indicates that there is not a remarkable change with the inclusion of solvent effects on the gas phase geometries. The differences of the C1-C4 distances at the six TSs are less than $0.06 \AA$. These results suggest that although the inclusion of solvent effects modifies substantially the energies, it does not change the electronic structure of the TSs (see later). 

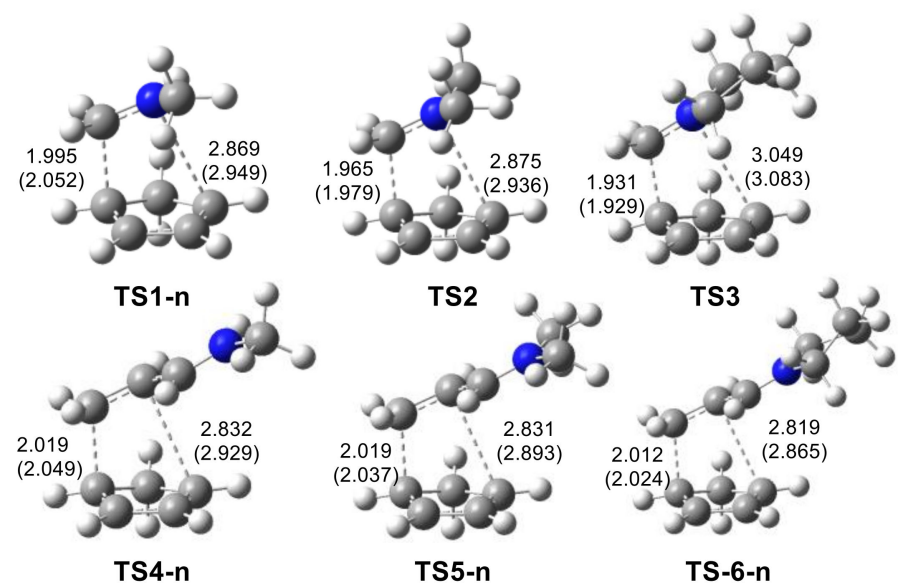

Figure 5. $\omega \mathrm{B} 97 \mathrm{XD} / 6-311 \mathrm{G}(\mathrm{d}, \mathrm{p})$ geometries of the endo TSs for DA reactions of iminium cations 6, 15-19 with $\mathrm{Cp}$ 1. The $\mathrm{C}-\mathrm{C}$ and $\mathrm{C}-\mathrm{N}$ distance are given in Angstroms. The gas phase distances are given in parentheses.

Herein, it is interesting to remark that unlike the P-DA reactions in which the TSs have a zwitterionic character resulting from the GEDT, at the TSs associated with I-DA reactions, both frameworks are positively charged. These behaviors have an important experimental consequence; in P-DA reactions, polar solvents accelerate the reactions by a better solvation of the zwitterionic TSs than reagents, whereas in I-DA reactions, solvent effects deaccelerate them by better solvation of the cationic reagents than TSs.

The ionic nature of these I-DA reactions was evaluated by computing the GEDT at the gas phase TSs. The computed GEDT values at the corresponding TSs, which flux from the nucleophilic Cp 1 to the superelectrophilic iminium cations, was: 0.40e (TS1-n), $0.43 \mathrm{e}$ (TS1-x), 0.43 e (TS2 and TS3), 0.37 e (TS4-n), 0.39 e (TS4-x), 0.38 (TS5-n and TS5-x), 0.38 e (TS6-n) and 0.37 (TS6-x). These very high values imply the high GEDT taking place at these I-DA reactions. Note that at the DA reactions involving neutral imines, the GEDT is lower than 0.15 e (see Section 1 in Supplementary Material). As expected, the GEDT values at the TSs associated with the I-DA reactions involving the most electrophilic iminium cations 6, 15 and 16, is slightly higher than those involving unsaturated iminium cations 17-18. The GEDT values at the TSs in acetonitrile are very close to those obtained in the gas phase; thus, the GEDT values of TS1-n and TS2 in acetonitrile are 0.41 e and 0.43 e, respectively. As expected, in these I-DA reactions, the electron density fluxed from $\mathrm{Cp} 1$ towards the iminium cations, these reactions being classified as the forward electron density flux (FEDF) [41].

As shown Figure 6, a good linear correlation between the GEDT and the asynchronicity at the TSs of the DA reaction of imines $\mathbf{1 3}$ and $\mathbf{1 4}$ and the I-DA reaction of cations 6, 15-19 can be established $\left(R^{2}=0.98\right)$. Thus, whereas the low polar DA reactions of neutral imines 13 and 14, GEDT $<0.15$ e, present low asynchronous TSs, $\Delta \mathrm{l}<0.2 \AA$, the I-DA reactions of iminium cations $6, \mathbf{1 5}-\mathbf{1 9}$ with GEDT $>0.37$ e present highly asynchronous TSs, $\Delta \mathrm{l}>0.8 \AA$. These behaviors are a consequence of fact that whereas low polar DA reactions of neutral imines are associated with low asynchronous four-center interactions, the I-DA reactions of non-symmetric iminium cations are associated with highly asynchronous twocenter interactions. These findings account for the decrease in the steric (Pauli) repulsion with the increase in the ionic character of the reaction. 


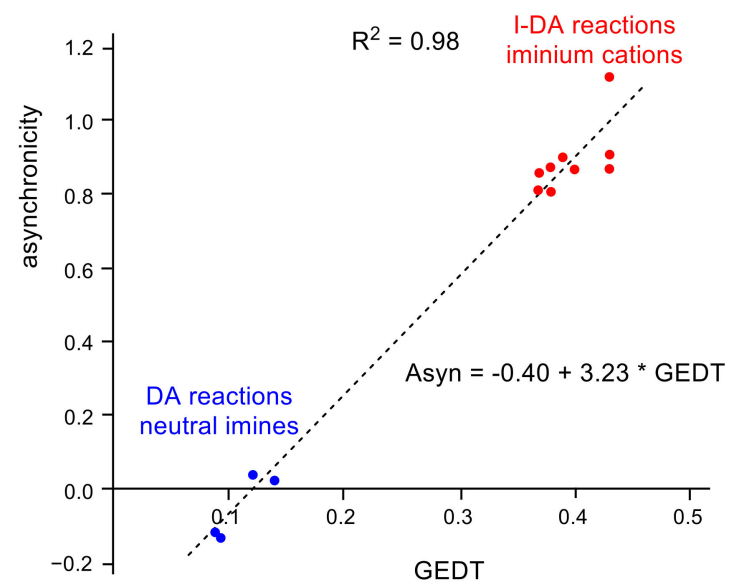

Figure 6. Plot of the asynchronicity, Asyn = (11-12) in Angstroms, vs. the GEDT, in e, taking place at the TSs associated with the DA reaction of non-symmetric imines and the I-DA reaction of nonsymmetric iminium cations.

\subsubsection{Study of the Regioselectivity in I-DA Reactions}

Due to the symmetry of Cp 1, these I-DA reactions do not have regioisomeric reaction paths. In order to analyze the regioselectivity in I-DA reactions, the reaction of iminium cation 6 with CpMe 20 was also considered (see Scheme 7). Due to the symmetry of iminium cation $\mathbf{6}$, only two competitive regioisomeric reaction paths, named ortho and meta, are feasible. The stationary points involved in this I-DA reaction are given in Scheme 7 , whereas the relative energies in acetonitrile are given in Table 3.

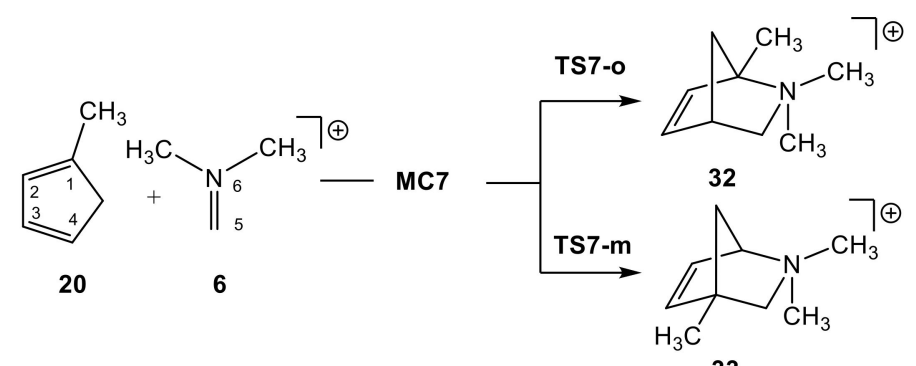

33

Scheme 7. I-DA reaction of iminium cations 6 with CpMe 20.

Table 3. $\omega B 97 X D / 6-311 G(\mathrm{~d}, \mathrm{p})$ relative electronic energies in acetonitrile, $\mathrm{kcal} \cdot \mathrm{mol}^{-1}$, of the stationary points involved in the I-DA reaction of iminium cation 6 with CpMe 20.

\begin{tabular}{ccc}
\hline MC7 & -7.5 \\
TS7-o & -0.8 \\
TS7-m & 2.7 \\
32 & -24.3 \\
33 & -24.1 \\
\hline
\end{tabular}

The relative energies of the TSs with respect to the separated reagents are -0.8 (TS7-o)

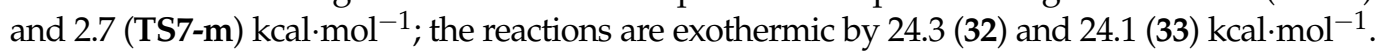
If the formation of MC7 is considered, the activation energies associated with the two regioisomeric reaction paths become 6.7 (TS7-o) and $10.2\left(\right.$ TS7-m) $\mathrm{kcal} \cdot \mathrm{mol}^{-1}$. Consequently, this I-DA reaction is completely regioselective, because TS7-m was found to be $3.5 \mathrm{kcal} \cdot \mathrm{mol}^{-1}$ above TS7-o. The activation energy associated with this I-DA reaction is $2.8 \mathrm{kcal} \cdot \mathrm{mol}^{-1}$ lower in energy than that involving Cp 1 via TS2, as a consequence of the more nucleophilic character of CpMe 20 than Cp 1 (see Tables 1 and 3).

The geometries of the two regioisomeric TSs associated with this I-DA reaction are given in Figure 7. The distances between the C1-C5 and C4-N6 interacting centers at 
the TSs of the I-DA reaction of iminium cation $\mathbf{6}$ with CpMe $\mathbf{2 0}$ in acetonitrile are 2.031 and $2.989 \AA$ at TS7-o, and 1.967 and $2.780 \AA$ at TS7-m. These distances indicate that these TSs are associated with highly asynchronous single bond formation processes which are controlled by the most electrophilic C 5 carbon of iminium cation 6 . The more favorable regioisomeric TS7-o is associated with the two-center interaction between the most electrophilic center of iminium cation $\mathbf{6}$ and the most nucleophilic center of CpMe 20, the non-substituted C4 carbon, a behavior anticipated by the analysis of the Parr functions (see Figure 4).
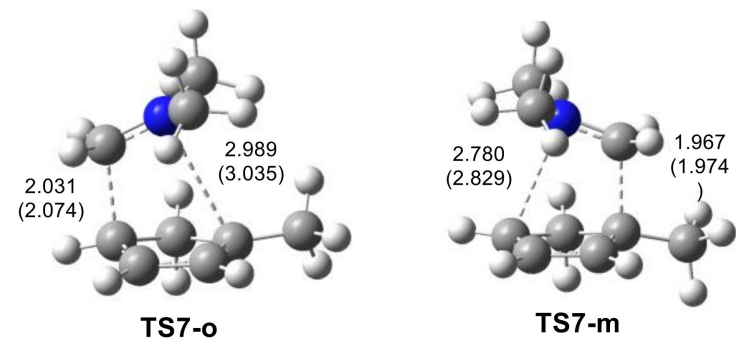

Figure 7. $\omega \mathrm{B} 97 \mathrm{XD} / 6-311 \mathrm{G}(\mathrm{d}, \mathrm{p})$ geometries of two regioisomeric TSs for I-DA reaction of iminium cation 6 with CpMe 20. The distances are given in Angstroms. The gas phase distances are given in parentheses.

At the two regioisomeric TSs, the GEDT taking place from the Cp framework to the iminium cation is $0.40 \mathrm{e}$ at TS7-o and $0.44 \mathrm{e}$ at TS7-m. These very high values point out the highly ionic character of this I-DA reaction. The GEDT at the more unfavorable TS7- $\mathrm{m}$ is higher than that at TS7-o as a consequence of the more advanced character of the former [42]. On the other hand, the high GEDT taking place at the TSs involving the non-symmetric iminium cation $\mathbf{6}$ accounts for the high asynchronicity found in this I-DA reaction.

\subsubsection{Thermodynamic Analysis of the I-DA Reaction of Iminium Cation 6 with Cp 1}

The thermodynamic data of the reaction of iminium cation 6 with $\mathrm{Cp} 1$, as a representative I-DA reaction of this series, were analyzed. Relative enthalpies and Gibbs free energies, computed at $25^{\circ} \mathrm{C}$ in acetonitrile, are given in Figure 8 .

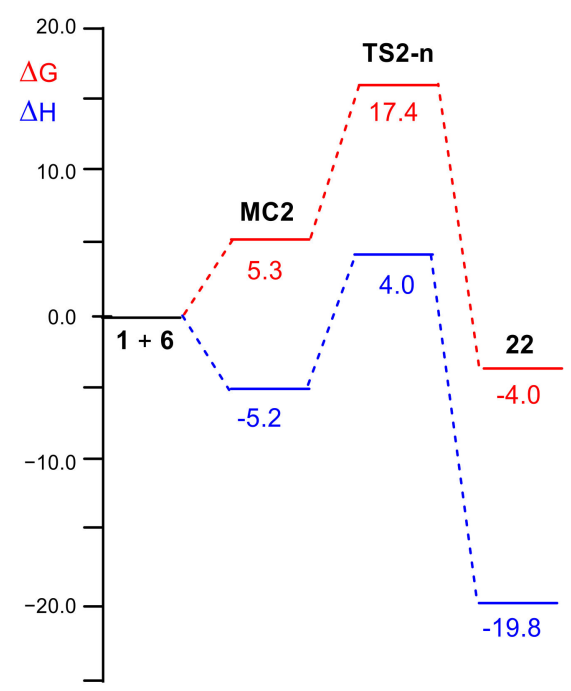

Figure 8. $\omega B$ 97XD/6-311G $(\mathrm{d}, \mathrm{p})$ enthalpy, in blue, $\Delta \mathrm{H}$ in $\mathrm{kcal} \mathrm{mol}^{-1}$ and Gibbs free energy, in red, $\Delta \mathrm{G}$ in $\mathrm{kcal} \mathrm{mol}^{-1}$ profiles in acetonitrile at $25^{\circ} \mathrm{C}$, for the I-DA reaction of iminium cation 6 with Cp 1 .

Inclusion of the thermal corrections with the electronic energies in acetonitrile decreases the relative enthalpies by between 1.1 and $4.2 \mathrm{kcal} \cdot \mathrm{mol}^{-1}$. The lower incidences 
take place in the relative enthalpy of TS2 which decreases by $1.1 \mathrm{kcal} \cdot \mathrm{mol}^{-1}$ with respect to the electronic energy in acetonitrile. The inclusion of the thermal correction and entropies in enthalpies increases the relative Gibbs free energies by between 10.5 and $15.9 \mathrm{kcal} \cdot \mathrm{mol}^{-1}$ as a consequence of the unfavorable activation entropy associated with these bimolecular processes, between -35.2 and $-53.3 \mathrm{cal} \cdot \mathrm{mol}^{-1} \cdot \mathrm{K}^{-1}$. Thus, the activation Gibbs free energy associated with the I-DA reaction of iminium cations $\mathbf{6}$ with $\mathrm{Cp} \mathbf{1}$ in acetonitrile rises to $17.4 \mathrm{kcal} \cdot \mathrm{mol}^{-1}$, while the formation of 22 becomes exergonic by only $-4.0 \mathrm{kcal} \cdot \mathrm{mol}^{-1}$. It is important to remark that although the formation of MC2 is an exothermic process, it is endergonic as a consequence of the unfavorable positive entropy associated with its formation (see Figure 8).

\subsection{Topological Analysis of the Bonding Changes along the I-DA Reactions}

MEDT establishes that the energy costs for bonding changes along a chemical reaction are responsible for its feasibility [9]. On the other hand, the comparative topological analysis of the electronic structures of TSs involved in the related reactions enables characterization of the similitudes or differences between them.

2.3.1. ELF Analysis of the C-C and N-C Single Bond Formation along the I-DA Reaction of Cp 1 with Iminium Cation 6

In order to understand the bonding changes along I-DA reactions, a BET study of the I-DA reaction of CP 1 with iminium cation $\mathbf{6}$ was performed. The detailed BET study of this I-DA reaction is given in Section 2 in the Supplementary Material. Some appealing conclusions can be obtained from this BET analysis: (i) the energy cost (EC) associated with formation of TS2, which is found at Phase III, $2.2 \mathrm{kcal} \cdot \mathrm{mol}^{-1}$, is mainly associated with the depopulation of the C5-N6 bonding region of the iminium moiety from 3.65 to 3.10 e. Thus, the activation energy of this I-DA reaction can be related to the EC associated with the rupture of the $\mathrm{C} 5=\mathrm{N} 6$ double bond of iminium cation 6; (ii) the bonding changes associated with this I-DA reaction are very similar to those found in P-DA reactions, but they are more advanced in I-DA reactions; note that in P-DA reactions the formation of the first $\mathrm{C}-\mathrm{C}$ single bond usually takes place afterwards to pass the TSs, whereas in this I-DA reaction, it takes place before to reach TS2. Note that at TS2, the C1-C5 single bond is already formed; (iii) although the C5 carbon of iminium cation 6 is the most electrophilic center of this molecule, the electron density coming from both the initial depopulation of C5-N6 bonding region and that of the GEDT is mainly accumulated at the N6 nitrogen first. Likewise, the bonding changes at the $\mathrm{Cp}$ framework are non-symmetric, and the changes in the $\mathrm{C} 1-\mathrm{C} 2$ bonding region are more advanced than those in the $\mathrm{C} 3-\mathrm{C} 4$ region. This behavior is a consequence of changes in electron density demanded for the formation of the first C1-C5 single bond; (iv) formation of the first $\mathrm{C} 1-\mathrm{C} 5$ single bond takes place at TS2, at a C-C distance of ca. $1.98 \AA$ and with an initial population of $0.76 \mathrm{e}$, by sharing the non-bonding electron density of two $\mathrm{C} 1$ and $\mathrm{C} 5$ pseudoradical centers (62:38) created from the rupture of the corresponding multiple bonds along the reaction path before reaching the TS [12]; (v) formation of the second C4-N6 single bond takes place at a C-N distance of ca. $2.11 \AA$ and with an initial population of $1.71 \mathrm{e}$, mostly through the donation of the non-bonding electron density of the N6 nitrogen developed from the rupture of the $\mathrm{C} 5=\mathrm{N} 6$ double bond of 6 to the C4 carbon (96:4); finally, (vi) this highly asynchronous I-DA reaction takes place through a marked non-concerted two-stage one-step mechanism [25], in which the formation of the second C4-N6 single bond takes place when the formation of the first C1-C5 bond is almost completed (94\%).

2.3.2. Comparative ELF Analysis of the TSs Involved in the P-DA Reaction of $\mathrm{Cp} 1$ with Imines 13 and 14, and Those Involved in the I-DA Reaction of Cp 1 with Iminium Cations 6 and 18

Finally, a comparative ELF topological analyses of the TSs involved in the P-DA reaction of Cp 1 with imine 13, TS8-n, unsaturated imine 14, TS9-n, and those involved in the I-DA reaction of Cp 1 with iminium cation 6, TS2, and unsaturated iminium cation 18, 
TS5-n, were performed in order understand the changes at the TSs of I-DA reactions with respect to those participating in P-DA reactions. The ELF attractors, with the most significant valence basin populations, are given in Figure 9.

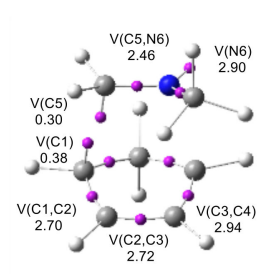

TS8-n

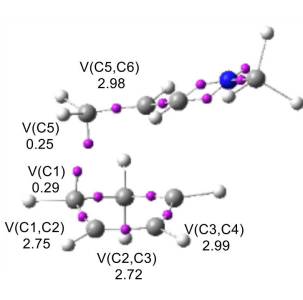

TS9-n

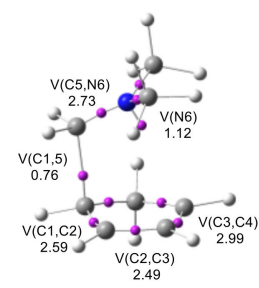

TS2

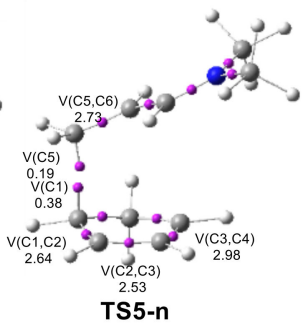

Figure 9. ELF basin attractor positions with the most significant valence basin populations of the TSs involved in the P-DA reaction of Cp 1 with imine 13, TS8-n, unsaturated imine 14, TS9-n, and those involved in the I-DA reaction of Cp 1 with iminium cation 6, TS2, and unsaturated iminium cation 18 , TS5-n.

The comparative analysis of the ELF of the polar TS8-n, TS9-n and ionic TS5-n indicates that they present great similarity. They show the presence of one $\mathrm{V}(\mathrm{C} 1)$ monosynaptic basin, integrating $0.38 \mathrm{e}, 0.29$ e and $0.38 \mathrm{e}$, respectively, and one $\mathrm{V}(\mathrm{C} 5)$ monosynaptic basin, integrating $0.30 \mathrm{e}, 0.25 \mathrm{e}$ and $0.19 \mathrm{e}$. These monosynaptic basins, which have been created along the reaction paths, are required for the subsequent $\mathrm{C} 1-\mathrm{C} 5$ single bond formation [12]. At these TSs, one $\mathrm{V}(\mathrm{C} 5, \mathrm{C}[\mathrm{N}] 6)$ disynaptic basin is observed, with populations of $2.46 \mathrm{e}$, $2.98 \mathrm{e}$ and $2.73 \mathrm{e}$, respectively, as a consequence of a slight depopulation of the $\mathrm{C}-\mathrm{C}[\mathrm{N}]$ bonding region. On the other hand, the $\mathrm{C} 1-\mathrm{C} 2-\mathrm{C} 3-\mathrm{C} 4$ bonding regions of these TSs are characterized by the presence of three disynaptic basins, $\mathrm{V}(\mathrm{C} 1, \mathrm{C} 2), \mathrm{V}(\mathrm{C} 2, \mathrm{C} 3)$ and $\mathrm{V}(\mathrm{C} 3, \mathrm{C} 4)$, integrating totals of $8.36 \mathrm{e}$ (TS8-n), 8.46 (TS9-n) and $8.15 \mathrm{e}$ (TS5-n), respectively. A significant part of the electron density lost in these frameworks, with respect to that of $\mathrm{Cp} \mathbf{1}$, $8.97 \mathrm{e}$, is involved in the formation of the $\mathrm{V}(\mathrm{C} 1)$ monosynaptic basins present at these TSs.

Different behavior is observed at TS2. Instead of the two $\mathrm{V}(\mathrm{C} 1)$ and $\mathrm{V}(\mathrm{C} 5)$ monosynaptic basins present at the other three TSs, a new $\mathrm{V}(\mathrm{C} 1, \mathrm{C} 5)$ disynaptic basin, integrating $0.76 \mathrm{e}$, is observed. The presence of this disynaptic basin indicates that at TS2, the formation of the new $\mathrm{C} 1-\mathrm{C} 5$ single bond has begun already. Considering that the formation of the $\mathrm{C}-\mathrm{C}$ single bonds takes place in the range of 2.0-1.9 $\AA$ [12], this different behavior of TS2 with respect to the other three TSs is a consequence of its more advanced character. On the order hand, the $\mathrm{C} 1-\mathrm{C} 2-\mathrm{C} 3-\mathrm{C} 4$ and $\mathrm{C} 5-\mathrm{C} 6$ bonding regions of TS2 are very similar to those at TS5-n.

From this ELF comparative analysis, it is possible to conclude that the four TSs belonging to P-DA reactions of imines and I-DA reactions of iminium cations present similar electronic structures. Considering the similarity of the ethylenic $\mathrm{C} 5-\mathrm{C}[\mathrm{N}] 6$ regions of these TSs, the very low activation energies associated with these I-DA reactions with respect to those of the P-DA reactions of imines 13 and 14, between 15 and $23 \mathrm{kcal} \cdot \mathrm{mol}^{-1}$, can be related to the high GEDT taking place at the TSs of these I-DA reactions which favor the changes of electron density along the corresponding reaction paths [43]. 


\subsubsection{Analysis of the Origin of the Regioselectivity in I-DA Reactions}

Many theoretical studies of cycloaddition reactions participating non-symmetric electrophilic species have established that the most favorable reaction path is that involving the two-center interaction between the most nucleophilic center of the nucleophile and the most electrophilic center of the electrophile species [39]. These favorable interactions, which facilitate the creation of the first $\mathrm{C}-\mathrm{C}$ single bonds, control the regio- and the chemoselectivity in polar reactions as well as the asynchronicity in single bond formation. In order to assert these finding in I-DA reactions, a topological analysis of the regioisomeric TS7-o and TS7- $\mathrm{m}$ was performed. The ELF attractors, with the most significant valence basin populations, are given in Figure 10.
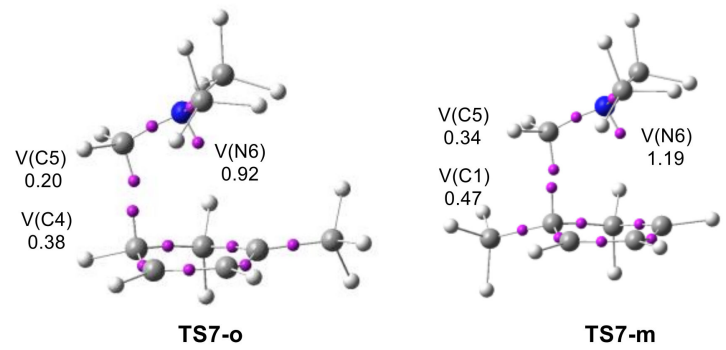

Figure 10. ELF basin attractor positions with the most significant valence basin populations of the regioisomeric TSs TS7-o and TS7-m involved in the I-DA reaction of CpMe 20 with iminium cation 6.

As can be seen, both regioisomeric TSs present a similar ELF structure. At the iminium cation framework, both TSs present two monosynaptic basins, V(C5) and V(N6), integrating 0.20 e and 0.92 at TS7-o and 0.34 and 1.19 e at TS7-m, respectively. Note that the populations of these monosynaptic basins are higher at the more unfavorable TS7-m because it is more advanced (see Figure 10). On the other hand, the populations of the $\mathrm{V}(\mathrm{C} 5, \mathrm{~N} 6)$ disynaptic basins at TS7-o, $2.91 \mathrm{e}$, and at TS7-m, $2.65 \mathrm{e}$, indicate that the C5-N6 bonding region of iminium cation 6 has experienced strong depopulations in these regions to create the $\mathrm{V}(\mathrm{N} 6)$ monosynaptic basin. Note that the population of this bonding region at iminium cation 6 is $3.48 \mathrm{e}$.

At the diene frameworks, whereas the most favorable TS7-o shows the presence of a $\mathrm{V}(\mathrm{C} 4)$ monosynaptic basin, integrating $0.38 \mathrm{e}$, at the most nucleophilic $\mathrm{C} 4$ carbon of CpMe 20 (see Figure 10), TS7-m presents a V(C1) monosynaptic basin, integrating 0.47 e at the substituted $\mathrm{C} 1$ carbon. On the order hand, the total electron density of the $\mathrm{C} 1-\mathrm{C} 2-\mathrm{C} 3-$ C4 bonding region of the $\mathrm{Cp}$ framework is $8.2 \mathrm{e}$ at TS7-o and $8.1 \mathrm{e}$ at TS7-m. Note that the total electron density of this bonding region at CpMe 20 is approximately $9.0 \mathrm{e}$.

Consequently, at the more favorable TS7-o, the V(C4) monosynaptic basin required for the asynchronous formation of the first $\mathrm{C} 4-\mathrm{C} 5$ single bond is created at the most electrophilic center of $\mathrm{CpMe} \mathrm{20}$, the $\mathrm{C} 4$ carbon, which corresponds to the carbon of $\mathrm{CpMe} 20$ that loses the least electron density along the GEDT process, a behavior anticipated by analysis of the nucleophilic $P_{k}^{-}$Parr functions (see Figure 4) [28].

\subsection{Origin of the Asynchronicity in I-DA Reactions}

Several studies have explored the origin of the high asynchronicity found in the single bond formation in polar cycloaddition reactions $[14,39]$. These studies have emphasized that the high asynchronicity is a consequence of the two-center interactions between the most nucleophilic center of the nucleophile and the most electrophilic center of the non-symmetrically substituted electrophilic species $[14,39]$. Note that many highly asynchronous TSs associated with P-DA reactions have been associated with Michael-type additions [42,44].

Highly P-DA and I-DA reactions are associated with two-center interactions; therefore, the two terminal centers that do not participate in the formation of the first single bond do not interact. Note that at highly asynchronous TSs, the C4 carbon of Cp 1 remains 
$\mathrm{sp}^{2}$-hybridized. As a consequence, along the approach of the superelectrophilic iminium cations to the nucleophilic $\mathrm{Cp}$ 1, three stereoisomeric TSs can be feasible in I-DA reactions: two gauche and one anti (see Figure 11). They are related to the $\mathrm{C} 2-\mathrm{C} 1-\mathrm{C} 5-\mathrm{C}(\mathrm{N}) 6$ dihedral angle at the TSs; thus, TS2, which is associated with the gauche 60 approach mode, presents a C2-C1-C5-N6 dihedral angle of 68.4 degrees.

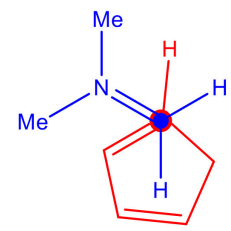

gauche -60

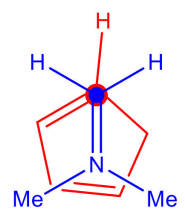

gauche 60

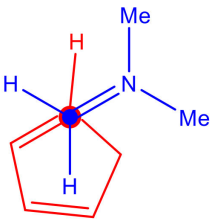

anti 180

Figure 11. Three feasible stereoisomeric reaction paths associated with the $C 4$ and $C 5$ two-center interaction in the I-DA reaction of $\mathrm{Cp} \mathbf{1}$ with iminium cation $\mathbf{6}$.

Thus, along the approach of $\mathrm{Cp} \mathbf{1}$ to iminium cation 6, it is possible to find the anti 180 TS, TS2-anti, in which the C2-C1-C5-N6 dihedral angle is -162.3 degrees (see Figure 12). The corresponding stepwise anti 180 reaction path is not competitive with the one-step gauche 60 path, because TS2-anti is found $3.6 \mathrm{kcal} \cdot \mathrm{mol}^{-1}$ above TS2. The GEDT at TS2anti, $0.48 \mathrm{e}$, is higher than that at TS2, $0.43 \mathrm{e}$, as a consequence of the shorter distance between the two frameworks at TS2-anti, $1.839 \AA$ A. Note that the GEDT depends on the electrophilic/nucleophilic character of the reagents and the distance between the two interacting frameworks [42].

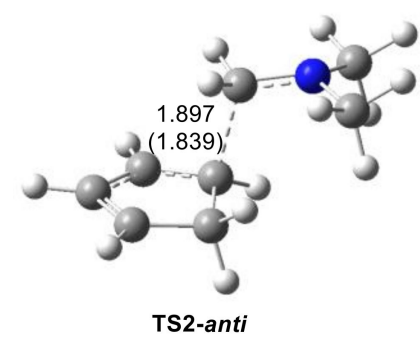

Figure 12. $\omega B 97 X D / 6-311 G(d, p)$ geometry of the stereoisomeric TS2-anti for the I-DA reaction of iminium cation 6 with $\mathrm{Cp}$ 1. The distances are given in Angstroms. The gas phase distance is given in parenthesis.

TS2 and TS2-anti are two stereoisomeric species. However, whereas TS2 is associated with a highly asynchronous TS, the stereoisomeric TS2-anti cannot be characterized as a highly asynchronous TS because it is associated with the first step of a stepwise IDA reaction. These stereoisomeric TSs are associated with a similar C1-C5 single bond formation process, yielding cycloadduct 22 (see Scheme 6). The unique relevant differences between both stereoisomeric TSs are the arrangement of the non-bonding electron density of the N6 nitrogen (see Figure S5 in Supplementary Material). Interestingly, whereas at the gauche 60 TS2 the two methyl groups of the iminium cation 6 are located above the hydrocarbon ring of $\mathrm{Cp}$ 1, at the anti 180 TS2-anti, they are farther away.

It appears that the favorable electronic interactions present between the non-bonding electron density created at the N6 nitrogen along the reaction path and the $\mathrm{C} 2-\mathrm{C} 3-\mathrm{C} 4$ allylic framework of $\mathrm{Cp}$ 1, which is positively charged, and not the steric repulsions associated with steric (Pauli) repulsions between the two interacting frameworks, which will be higher at the more favorable gauche 60 TS2 than at anti 180 TS2-anti, such has been suggested [29], will be responsible for the higher stability of TS2 than TS2-anti. Consequently, it appears that steric repulsions do not control anything.

It can be concluded that the observed decrease in the steric repulsion with the increase in the nucleophilic/electrophilic interactions is a consequence of the increase in the 
asynchronicity resulting from an increase in the favorable two-center interactions taking place at the polar and ionic TSs involving asymmetric electrophilic species caused by the GEDT. Therefore, the reduction in the steric (Pauli) repulsions characterized by Bickelhaup et al. after performing EDA [29], is a consequence of the increase in the asynchronicity, which increases the distance between the ends of the two interacting frameworks, and never vice versa.

\section{Conclusions}

The behaviors of the I-DA reactions have been investigated within the MEDT at the $\omega \mathrm{B} 97 \mathrm{XD} / 6-311 \mathrm{G}(\mathrm{d}, \mathrm{p})$ computational level. To this end, the P-DA reactions of two imines 13 and 14, and the I-DA reactions of six iminium cations 6, 13-19 with Cp 1 have been investigated.

ELF topological analysis of these aza derivatives shows that the double bonds of both imines and iminium cations are very similar. Interestingly, the populations at the double bonds of iminium cations are slightly higher than at imines as a consequence of the reorganization of the non-bonding electron density of the $\mathrm{N}$ nitrogen present in imines.

The superelectrophilic character of iminium cations, $\omega>8.20 \mathrm{eV}$, accounts for the high reactivity of these species with respect to that of the corresponding neutral imines, $\omega<1.31 \mathrm{eV}$, participating in P-DA reactions. Analysis of the electrophilic $P_{k}^{+}$Parr functions at the iminium cations characterizes the $\mathrm{C} 5$ carbons as the most electrophilic center of these species, thus accounting for the high regioselectivity and high asynchronicity found in these I-DA reactions.

The activation energies of the I-DA reactions were found to be between 13 and $20 \mathrm{kcal} \cdot \mathrm{mol}^{-1}$ lower in energy than those associated with the P-DA reactions of neutral imines. These I-DA reactions are low endo selective as a consequence of the cationic character of the TSs, but highly regioselective.

Unlike P-DA reactions, polar solvents have a poor effect on the relative energies, and an unappreciable effect on the geometries. Interestingly, in solution, the activation energies increase slightly as a consequence of the better solvation of the iminium cations than TSs.

ELF topological analysis of the bonding changes along the I-DA reaction of iminium cation 6 with $\mathrm{Cp} 1$ shows that $\mathrm{C}-\mathrm{C}$ single bond formation takes place at TS2 at a C$\mathrm{C}$ distance of ca. $1.98 \AA$, and with an initial population of $0.76 \mathrm{e}$, by sharing the nonbonding electron density of two $C 1$ and C5 pseudoradical centers (62:38) created from the rupture of the corresponding multiple bonds along the reaction path before reaching the TS. These centers correspond with the most electrophilic C5 carbon of iminium cation $\mathbf{6}$, and to the most nucleophilic $\mathrm{C} 1$ carbon of $\mathrm{Cp} 1$, behaviors anticipated by analysis of the Parr functions. Interestingly, the pattern found for the $\mathrm{C}-\mathrm{C}$ bond formation in this I-DA reaction is very similar to that found in N-DA and P-DA reactions [12].

Finally, an ELF comparative analysis of the TSs involved in the P-DA reactions of imines and those participating in the I-DA reactions of iminium cations showed that there are a great similarity in the bonding changes in both polar and ionic TSs. The main difference was found in the GEDT taking place in ionic reactions, higher than $0.37 \mathrm{e}$; the high GEDT taking place in I-DA reactions markedly decreased the activation energies to diminish the ECs associated with the changes in electron density required to determine the electronic structure of the TSs.

Well established concepts in organic chemistry, such as electrophilicity and nucleophilicity, are used within MEDT to explain and even to predict the chemical reactivity of iminium cations participating in I-DA reactions. On the other hand, the high GEDT taking place in the cationic TSs explains the low endo selectivity and low solvent effects found in I-DA reactions. The present MEDT study establishes that the high GEDT taking place at the TSs of the I-DA reactions resulting from the superelectrophilic character of the iminium cations is responsible for the features of these types of DA reactions. This contrasts with 
the steric (Pauli) repulsions origin proposed recently by Bickelhaup et al. [29,45] based on an ASM analysis, which is markedly different from the MEDT.

\section{Computational Details}

DFT calculations were performed using the hybrid $\omega$ B97X-D function [46], which includes long range exchange (denoted by $\mathrm{X}$ ) correction as well as the semiclassical Londondispersion correction (indicated by suffix-D). The standard 6-311G $(\mathrm{d}, \mathrm{p})$ basis set was used [47], which includes d-type polarization for second-row elements and p-type polarization functions for hydrogen atoms. The Berny method was used in optimizations [48,49]. Only one imaginary frequency characterized all studied TSs. The intrinsic reaction coordinate (IRC) paths [50] were explored to find the unique connections given between the TSs and the minimum stationary points [51,52]. Solvent effects of acetonitrile were considered by full optimization of the gas phase structures at the same computational level using the polarizable continuum model [53,54] (PCM) in the framework of the self-consistent reaction field [55-57] (SCRF).

The GEDT [12] values were estimated by an NPA [34,35], using the equation GEDT $(\mathrm{f})=\Sigma_{\mathrm{q} \in \mathrm{f}} \mathrm{q}$, where $\mathrm{q}$ is the charge at atoms of a framework $(f)$ at the TSs. CDFT reactivity indices $[18,19]$ were calculated through the equations given in [19].

All calculations were carried out with the Gaussian 16 suite of programs [58]. The topology of the ELF [33] of the $\omega \mathrm{B} 97 \mathrm{XD} / 6-311 \mathrm{G}(\mathrm{d}, \mathrm{p})$ monodeterminantal wave functions was assessed using the TopMod [59] package with a cubical grid of step size of $0.1 \mathrm{Bohr}$. The GaussView program [60] was used to visualize molecular geometries of all the systems as well as the position of the ELF basin attractors. The ELF localization domains at an isovalue of 0.75 a.u. were obtained with Paraview software [61,62].

Supplementary Materials: The following are available online. Study of the DA reactions of imines 13 and 14 with $C p 1$. BET study of the I-DA reaction of iminium cation 6 with $C p 1$. Figure with the plot of the asynchronicity vs. the GEDT taking place at eight of the TSs associated with the P-DA and I-DA reactions given in Scheme 1. Figure with the wB97XD/6-311G $(\mathrm{d}, \mathrm{p})$ geometries of the exo TSs of the I-DA reactions of iminium cations 6, 17-19 with Cp 1. Figure with the wB97XD/6$311 \mathrm{G}(\mathrm{d}, \mathrm{p})$ localization domains and ELF basin attractor positions of the gauche $60^{\circ} \mathrm{TS} 2$, the anti TS2-anti involved in the I-DA reaction of Cp $\mathbf{1}$ with iminium cation $\mathbf{6}$, and the point of the IRC from TS2 in which the C4-C5 distance is similar to that at the anti TS2-anti. Table with $\omega B 97 X D / 6-$ $311 \mathrm{G}(\mathrm{d}, \mathrm{p})$ electronic energies, in acetonitrile, of the stationary points involved in the I-DA reactions of iminium cations 6, 15-19 with Cp 1. Table with the $\omega \mathrm{B} 97 \mathrm{XD} / 6-311 \mathrm{G}(\mathrm{d}, \mathrm{p})$ thermodynamic data of the stationary points involved in the I-DA reactions of imine cation 6 with $\mathrm{Cp} 1$.

Author Contributions: L.R.D. headed the subject, wrote the manuscript, and performed the calculations; M.R.-G. performed the calculations and wrote the manuscript and M.J.A. performed the calculations and wrote the manuscript. All authors have read and agreed to the published version of the manuscript.

Funding: This research received funding from the Ministry of Economy and Competitiveness (MINECO) of the Spanish Government, project PID2019-110776GB00 (AEI/FEDER, UE), and the European Union's Horizon 2020 research and innovation program under the Marie Skłodowska-Curie grant agreement No. 84618.

Institutional Review Board Statement: Not applicable.

Informed Consent Statement: Not applicable.

Data Availability Statement: Data can be made available upon written request to the corresponding author.

Acknowledgments: L.R.D. and M.J.A. acknowledge the Ministry of Economy and Competitiveness (MINECO) of the Spanish Government, and M.R.-G. acknowledge the European Commission for financial support. 
Conflicts of Interest: There are no conflict to declare.

Sample Availability: Not applicable.

\section{References}

1. Diels, O.; Alder, K. Synthesen in der hydroaromatischen Reihe. Justus Liebigs Ann. Chem. 1928, 460, 98-122. [CrossRef]

2. Carruthers, W. Some Modern Methods of Organic Synthesis, 2nd ed.; Cambridge University Press: Cambridge, UK, 1978.

3. Carruthers, W. Cycloaddition Reactions in Organic Synthesis; Pergamon: Oxford, UK, 1990.

4. Woodward, R.B.; Hoffmann, R. The Conservation of the Orbital Symmetry. Angew. Chem. Int. Ed. Engl. 1969, 8, 781-853. [CrossRef]

5. Houk, K.N.; Gonzalez, J.; Li, Y. Pericyclic Reaction Transition States: Passions and Punctilios, 1935-1995. Acc. Chem. Res. 1995, 28, 81-90. [CrossRef]

6. Krokidis, X.; Noury, S.; Silvi, B. Characterization of Elementary Chemical Processes by Catastrophe Theory. J. Phys. Chem. A 1997, 101, 7277-7282. [CrossRef]

7. Berski, S.; Andrés, J.; Silvi, B.; Domingo, L.R. The Joint Use of Catastrophe Theory and Electron Localization Function to Characterize Molecular Mechanisms. A Density Functional Study of the Diels-Alder Reaction between Ethylene and 1,3Butadiene. J. Phys. Chem. A 2003, 107, 6014-6024. [CrossRef]

8. Domingo, L.R.; Ríos-Gutiérrez, M.; Silvi, B.; Pérez, P. The Mysticism of Pericyclic Reactions: A Contemporary Rationalisation of Organic Reactivity Based on Electron Density Analysis. Eur. J. Org. Chem. 2018, 2018, 1107-1120. [CrossRef]

9. Domingo, L.R. Molecular electron density theory: A modern view of reactivity in organic chemistry. Molecules 2016, 21, 1319. [CrossRef] [PubMed]

10. Fukui, K. Molecular Orbitals in Chemistry, Physics, and Biology; Academic Press: New York, NY, USA, 1964.

11. Dewar, M.J.S. Aromaticity and Pericyclic Reaction. Angew. Chem. Int. Ed. 1971, 10, 761-776. [CrossRef]

12. Domingo, L.R. A new C-C bond formation model based on the quantum chemical topology of electron density. RSC Adv. 2014, 4 , 32415-32428. [CrossRef]

13. Domingo, L.R.; Arnó, M.; Andrés, J. Influence of Reactant Polarity on the Course of the Inverse-Electron-Demand Diels-Alder Reaction. A DFT Study of Regio- and Stereoselectivity, Presence of Lewis Acid Catalyst, and Inclusion of Solvent Effects in the Reaction between Nitroethene and Substituted Ethenes. J. Org. Chem. 1999, 64, 5867-5875.

14. Domingo, L.R.; Aurell, M.J.; Perez, P.; Contreras, R. Origin of the synchronicity on the transition structures of polar Diels-Alder reactions. Are these reactions [4 +2] processes? J. Org. Chem. 2003, 68, 3884-3890. [CrossRef]

15. Domingo, L.R.; Sáez, J.A. Understanding the mechanism of polar Diels-Alder reactions. Org. Biomol. Chem. 2009, 7, 3576-3583. [CrossRef] [PubMed]

16. Parr, R.G.; Szentpaly, L.V.; Liu, S. Electrophilicity index. J. Am. Chem. Soc. 1999, 121, 1922-1924. [CrossRef]

17. Domingo, L.R.; Chamorro, E.; Pérez, P. Understanding the reactivity of captodative ethylenes in polar cycloaddition reactions. A theoretical study. J. Org. Chem. 2008, 73, 4615-4624. [CrossRef]

18. Parr, R.G.; Yang, W. Density Functional Theory of Atoms and Molecules; Oxford University Press: Oxford, UK, 1989.

19. Domingo, L.R.; Ríos-Gutiérrez, M.; Pérez, P. Applications of the conceptual density functional indices to organic chemistry reactivity. Molecules 2016, 21, 748. [CrossRef] [PubMed]

20. Terrier, F.; Sebban, M.; Goumont, R.; Hallé, J.C.; Moutiers, G.; Cangelosi, I.; Buncel, E. Dual Behavior of 4-Aza-6-nitrobenzofuroxan. A Powerful Electrophile in Hydration and $\sigma$-Complex Formation and a Potential Dienophile or Heterodiene in Diels-Alder Type Reactions. J. Org. Chem. 2000, 65, 7391-7398. [CrossRef] [PubMed]

21. Domingo, L.R.; Pérez, P. The Lithium Cation Catalysed Benzene Diels-Alder reaction. Insights on the Molecular Mechanism within the Molecular Electron Density Theory. J. Org. Chem. 2020, 85, 13121-13132. [CrossRef]

22. Mayr, H.; Ofial, A.R.; Sauer, J.; Schmied, B. [2+ +4$]$ Cycloadditions of Iminium Ions-Concerted or Stepwise Mechanism of Aza Diels-Alder Reactions? Eur. J. Org. Chem. 2000, 2000, 2013-2020. [CrossRef]

23. Hedberg, C.; Pinho, P.; Roth, P.; Andersson, P.G. Diels-Alder Reaction of Heterocyclic Imine Dienophiles. J. Org. Chem. 2000, 65, 2810-2812. [CrossRef]

24. Domingo, L.R. A Theoretical Study of the Molecular Mechanism of the Reaction between N,N-Dimethylmethyleneammonium Cation and Cyclopentadiene. J. Org. Chem. 2001, 66, 3211-3214. [CrossRef]

25. Domingo, L.R.; Sáez, J.A.; Zaragozá, R.J.; Arnó, M. Understanding the Participation of Quadricyclane as Nucleophile in Polar [2 $\sigma$ $+2 \sigma+2 \pi$ ] Cycloadditions toward Electrophilic $\pi$ Molecules. J. Org. Chem. 2008, 73, 8791-8799. [CrossRef]

26. Domingo, L.R.; Oliva, M.; Andrés, J. A theoretical study of the reaction between cyclopentadiene and protonated imine derivatives: A shift from a concerted to a stepwise molecular mechanism. J. Org. Chem. 2001, 66, 6151-6157. [CrossRef] [PubMed]

27. Domingo, L.R.; Peréz, P. A Quantum Chemical Topological Analysis of the C-C Bond Formation in Organic Reactions Involving Cationic Species. Phys. Chem. Chem. Phys. 2014, 16, 14108-14115. [CrossRef]

28. Domingo, L.R.; Perez, P.; Sáez, J.A. Understanding the local reactivity in polar organic reactions through electrophilic and nucleophilic Parr functions. RSC Adv. 2013, 3, 1486-1494. [CrossRef]

29. Vermeeren, P.; Hamlin, T.A.; Fernandez, I.; Bickelhaupt, F.M. Origin of rate enhancement and asynchronicity in iminium catalyzed Diels-Alder reactions. Chem. Sci. 2020, 11, 8105-8112. [CrossRef] 
30. Van Zeist, W.-J.; Bickelhaupt, F.M. The activation strain model of chemical reactivity. Org. Biomol. Chem. 2010, 8, 3118-3127. [CrossRef]

31. Bickelhaupt, F.M. Understanding reactivity with Kohn-Sham molecular orbital theory: E2-SN2 mechanistic spectrum and other concepts. J. Comput. Chem. 1999, 20, 114-128. [CrossRef]

32. Domingo, L.R.; Aurell, M.J.; Perez, P.; Sáez, J.A. Understanding the Origin of the Asynchronicity in Bond-Formation in Polar Cycloaddition Reactions. A DFT Study of the 1,3-Dipolar Cycloaddition Reaction of Carbonyl Ylides with 1,2-Benzoquinones. RSC Adv. 2012, 2, 1334-1342. [CrossRef]

33. Becke, A.D.; Edgecombe, K.E. A simple measure of electron localization in atomic and molecular-systems. J. Chem. Phys. 1990, 92, 5397-5403. [CrossRef]

34. Reed, A.E.; Weinstock, R.B.; Weinhold, F. Natural population analysis. J. Chem. Phys. 1985, 83, 735-746. [CrossRef]

35. Reed, A.E.; Curtiss, L.A.; Weinhold, F. Intermolecular interactions from a natural bond orbital, donor-acceptor viewpoint. Chem. Rev. 1988, 88, 899-926. [CrossRef]

36. Pérez, P.; Domingo, L.R.; Aizman, A.; Contreras, R. The Electrophilicity Index in Organic Chemistry. In Theoretical Aspects of Chemical Reactivity; Toro-Labbe, A., Ed.; Elsevier: Amsterdam, The Netherlands, 2007; Volume 9, pp. $139-201$.

37. Domingo, L.R.; Pérez, P. The Nucleophilicity N Index in Organic Chemistry. Org. Biomol. Chem. 2011, 9, 7168-7175. [CrossRef] [PubMed]

38. Parr, R.G.; Pearson, R.G. Absolute hardness: Companion parameter to absolute electronegativity. J. Am. Chem. Soc. 1983, 105, 7512-7516. [CrossRef]

39. Aurell, M.J.; Domingo, L.R.; Perez, P.; Contreras, R. A theoretical study on the regioselectivity of 1,3-dipolar cycloadditions using DFT-based reactivity indexes. Tetrahedron 2004, 60, 11503-11509. [CrossRef]

40. Hammond, G.S. A Correlation of Reaction Rates. J. Am. Chem. Soc. 1955, 77, 334-338. [CrossRef]

41. Domingo, L.R.; Ríos-Gutiérrez, M.; Pérez, P. A Molecular Electron Density Theory Study of the Reactivity of Tetrazines in Aza-Diels-Alder Reactions. RSC Adv. 2020, 10, 15394-15405. [CrossRef]

42. Domingo, L.R.; Ríos-Gutiérrez, M.; Pérez, P. Unveiling the Lewis Acid Catalysed Diels-Alder Reactions Through the Molecular Electron Density Theory. Molecules 2020, 25, 2535. [CrossRef]

43. Domingo, L.R.; Ríos-Gutiérrez, M.; Pérez, P. How does the global electron density transfer diminish activation energies in polar cycloaddition reactions? A Molecular Electron Density Theory study. Tetrahedron 2017, 73, 1718-1724. [CrossRef]

44. Domingo, L.R.; Jones, R.A.; Picher, M.T.; Sepulveda-Arques, J. Theoretical study of the reaction of dimethyl acetylenedicarboxilate with 1-methyl-2-(1-substituted vinyl) pyrroles. Tetrahedron 1995, 51, 8739-8748. [CrossRef]

45. Hamlin, T.A.; Bickelhaupt, F.M.; Fernández, I. The Pauli Repulsion-Lowering Concept in Catalysis. Acc. Chem. Res. 2021, 54, 1972-1981. [CrossRef]

46. Chai, J.-D.; Head-Gordon, M. Long-range corrected hybrid density functionals with damped atom-atom dispersion corrections. Phys. Chem. Chem. Phys. 2008, 10, 6615-6620. [CrossRef]

47. Hehre, M.J.; Radom, L.; Schleyer, P.V.R.; Pople, J. Ab initio Molecular Orbital Theory; Wiley: New York, NY, USA, 1986.

48. Schlegel, H.B. Optimization of equilibrium geometries and transition structures. J. Comput. Chem. 1982, 3, 214-218. [CrossRef]

49. Schlegel, H.B. Modern Electronic Structure Theory; Yarkony, D.R., Ed.; World Scientific Publishing: Singapore, 1994.

50. Fukui, K. Formulation of the reaction coordinate. J. Phys. Chem. 1970, 74, 4161-4163. [CrossRef]

51. González, C.; Schlegel, H.B. Reaction path following in mass-weighted internal coordinates. J. Phys. Chem. 1990, 94, 5523-5527. [CrossRef]

52. González, C.; Schlegel, H.B. Improved algorithms for reaction path following: Higher-order implicit algorithms. J. Chem. Phys. 1991, 95, 5853-5860. [CrossRef]

53. Tomasi, J.; Persico, M. Molecular interactions in solution: And overview of methods based on continuous distributions of the solvent. Chem. Rev. 1994, 94, 2027-2094. [CrossRef]

54. Simkin, B.Y.; Sheikhet, I.I. Quantum Chemical and Statistical Theory of Solutions-Computational Approach; Ellis Horwood: London, UK, 1995.

55. Cossi, M.; Barone, V.; Cammi, R.; Tomasi, J. Ab initio study of solvated molecules: A new implementation of the polarizable continuum model. Chem. Phys. Lett. 1996, 255, 327-335. [CrossRef]

56. Cances, E.; Mennucci, B.; Tomasi, J. A new integral equation formalism for the polarizable continuum model: Theoretical background and applications to isotropic and anisotropic dielectrics. J. Chem. Phys. 1997, 107, 3032-3041. [CrossRef]

57. Barone, V.; Cossi, M.; Tomasi, J. Geometry optimization of molecular structures in solution by the polarizable continuum model. J. Comput. Chem. 1998, 19, 404-417. [CrossRef]

58. Frisch, M.J.; Trucks, G.W.; Schlegel, H.B.; Scuseria, G.E.; Robb, M.A.; Cheeseman, J.R.; Scalmani, G.; Barone, V.; Petersson, G.A.; Nakatsuji, H.; et al. Gaussian 16, Revision A.03; Gaussian, Inc.: Wallingford, CT, USA, 2016.

59. Noury, S.; Krokidis, X.; Fuster, F.; Silvi, B. Computational tools for the electron localization function topological analysis. Comput. Chem. 1999, 23, 597-604. [CrossRef]

60. Dennington, R.; Keith, T.A.; Millam, J.M. GaussView, Version 6.0; Semichem Inc.: Shawnee Mission, KS, USA, 2016.

61. Ahrens, J.; Geveci, B.; Law, C. ParaView: An End-User Tool for Large Data Visualization, Visualization Handbook; Elsevier: Amsterdam, The Netherlands, 2005. [CrossRef]

62. Ayachit, U. The ParaView Guide: A Parallel Visualization Application; Kitware: Clifton Park, NY, USA, 2015; ISBN 978-1930934306. 